\title{
Review
}

\section{A flexible symmetry-preserving Galerkin/POD reduced order model applied to a convective instability problem}

\author{
Francisco Pla $^{\mathrm{a}}$ Henar Herrero ${ }^{\mathrm{a}}$, José M. Vega ${ }^{\mathrm{b}}$ \\ ${ }^{a}$ Departamento de Matemáticas, Facultad de Ciencias y Tecnologías Químicas, Universidad de Castilla-La Mancha, 13071 Ciudad Real, Spain \\ ${ }^{\mathrm{b}}$ Escuela de Ingeniería Aeronáutica y del Espacio, Universidad Politécnica de Madrid, 28040 Madrid, Spain
}

\begin{abstract}
A B S T R A C T
A flexible Galerkin method based on proper orthogonal decomposition (POD) is described to construct the bifurcation diagram, as the Rayleigh number $R$ is varied, in the Rayleigh-Bênard convection in a rectangular box for large Prandtl number. The bifurcation diagram is approximated using the POD modes resulting from unconverged snapshots for just one specific value of $R$, calculated in either Newton iterations or time-dependent runs converging to steady states. Moreover, the selection of the specific value of $R$ is quite flexible. In addition, a horizontal reflection symmetry is taken into account to construct a symmetry-preserving Galerkin system. The resulting un-symmetric and symmetric low-dimensional systems are combined with a basic continuation method, which provide the bifurcation diagram at a quite low computational cost.
\end{abstract}

\section{Introduction}

The study of instabilities and bifurcations for partial differential equations and systems is of paramount scientific interest and is becoming more and more necessary in industrial problems as well, due to the increasing need to take nonlinearity into account. The difficulty is that calculating bifurcation diagrams requires solving the problem for a large number of parameter values. Furthermore, each of these individual calculations may be very computationally expensive if, e.g., fluid dynamics calculations are involved. Thus, huge computational resources (CPU time and memory) may be needed using standard numerical solvers $[11,48,53]$ and continuation methods $[1,33]$. The difficulty may 
be overcome replacing standard numerics by reduced order modeling, such as reduced basis $[41,35,16,32]$ and Galerkin/POD methods. The latter methods have been used to treat both steady $[29,2,3,7,4]$ and unsteady systems $[36,49]$, in both purely scientific $[37,28,13,46,47,45]$ and industrial $[17,31,30,52,10]$ applications. Reduced modeling relies on the observation that if the governing evolution equations are dissipative, the large time behavior of the system is contained in a finite dimensional inertial manifold $[18,25,40]$, which is frequently low dimensional $[19,34]$. The application of POD to an appropriate set of snapshots calculated by a standard numerical solver (NS) provides a linear manifold that contains the inertial manifold, which can be used to obtain POD-based reduced order models (ROMs). For fluid flow problems, several strategies have been tried using both reduced basis and POD/Galerkin methods $[16,26,28,32,41]$, which resulted in various methodological/computational improvements.

In principle, when applied to bifurcation problems, both reduced basis and Galerkin/POD methods require a preprocess, where some snapshots are calculated using a standard numerical solver. The snapshots are usually $[24,23]$ solutions of the same type as those that are to be approximated (e.g., steady states) and are calculated for various (say, $N$ ) representative values of the bifurcation parameter. Since each snapshot is a converged steady state of the nonlinear problem, the preprocess may be quite computationally expensive if $N$ is large, and somehow makes the method an interpolation method, able to calculate the solutions for intermediate parameter values using information from the specific parameter values where the snapshots were calculated. In any event, $N$ must be somewhat larger than the number of degrees of freedom of the low-dimensional model. Since the computational cost of the preprocess depends critically on $N$, some effort has been devoted in the references quoted above to appropriately select the specific values of the bifurcation parameter for which the snapshots are calculated.

The approach in this paper is fairly different. It comes from observation that transient behavior contains more useful information to calculate the converged states than the converged states themselves. In other words, if unconverged states in temporal runs for a few particular values of the bifurcation parameter are used as snapshots, then the resulting POD modes are useful to calculate the converged states for other values of the bifurcation parameter. This property was emphasized and illustrated [50] in some academic problems such as the one-dimensional Fisher and Ginzburg-Landau equations, where the whole bifurcation diagram was constructed using as snapshots the unconverged states in a temporal run for just one value of the bifurcation parameter, which moreover was selected somewhat arbitrarily. Note that the preprocess consisted in the calculation of just one point of the bifurcation diagram, meaning that the preprocess was quite computationally inexpensive. The reader is referred to [50] for other somewhat surprising/encouraging consequences of all these. Note that, in contrast to the method outlined in the former paragraph, this strategy can be seen as an extrapolation because the whole bifurcation diagram is constructed using information from only one point in the diagram.

Building upon this property and former ideas to construct adaptive time-dependent ROMs [36,49], an adaptive method can be constructed [51] that turns out to be quite efficient to construct bifurcation diagrams for the one-dimensional complex GinzburgLandau equation. Even though this equation is a well-known paradigm for pattern formation [5] that exhibits quite complex dynamics, a question remains on whether these ideas work equally well in higher dimensional fluid dynamics problems, which are more demanding from several points of view. The present paper is a step in this direction, considering the two-dimensional Rayleigh-Bénard convection at large Prandtl number. Thermal convection is known to be the driving force of many physical phenomena and industrial applications. Standard Rayleigh-Bénard convection in a horizontal layer heated from below is a fairly simplified model for atmospheric convection [8], in which the conductive quiescent state becomes unstable for a critical vertical temperature gradient, measured by the Rayleigh number $R$. Beyond this threshold, a convective motion sets in that suffers further transitions as the $R$ is further increased. The large Prandtl number limit is of interest in geophysics [15,38], and has been analyzed in [33], where the bifurcation diagram was calculated for the thermal convection in a two-dimensional box. Anticipating the main conclusion in this paper for this problem, the whole bifurcation diagram for $0 \leq R \leq 3000$ will be approximated using ROMs based on unconverged snapshots calculated at just one value of the Rayleigh number $R$. Moreover, this value can be chosen somewhat arbitrarily. Let us mention here that this same test problem was considered in [23] using a more standard method based on reduced basis selecting the snapthots as steady states at various values of the parameter $R$, ordered in a greedy way.

The test problem exhibits an exact horizontal reflection symmetry. Symmetries play an important role in bifurcation problems, in particular, in fluid dynamics $[21,14]$. Some of the bifurcations in systems exhibiting symmetries are associated with symmetry breaking (or loss) and some others preserve the symmetries, producing symmetric states. Reduced models can be constructed such that they inherit the symmetries of the problem, as first done twenty years ago in a seminal paper [6]. The idea was further developed by Shah and Sorensen [42-44], but has not been otherwise exploited in the literature (to our knowledge) to the extend this topic deserves. Here, we shall construct a symmetrypreserving reduced model of the test problem that will be considered.

With these ideas in mind, the article is organized as follows. The test problem is formulated in Section 2, where the description of the physical configuration is provided, along with the basic equations, boundary conditions, and the standard numerical solver that is to be used to calculate the snapshots. POD is thoroughly discussed in Section 3, where an improved version of this method is described (yielding very good approximations of POD modes associated with very small singular values) and the combination with the reflection symmetry is considered. Several ROMs, ignoring and taking the symmetry into account, and using snapshots calculated from temporal runs and the Newton method, are constructed in Sections 4 and 5, where a simple continuation for the Galerkin systems is presented, along with the application to the thermal convection problem. Finally, several concluding remarks are considered in Section 6.

\section{Formulation of the problem}

As sketched in Fig. 1, a two-dimensional fluid layer of depth $d$ is considered that is confined between a lower solid plate and an

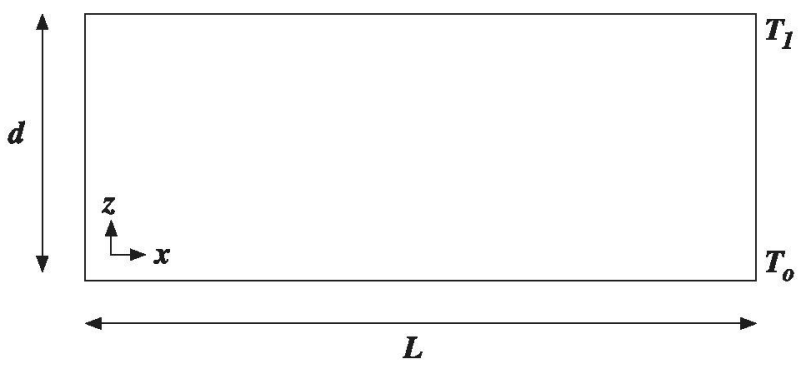

Fig. 1. Sketch of the thermal convection system. 
upper non-deformable free surface, where the temperatures are imposed to be $T_{0}$ and $T_{1}$, respectively, with $\Delta T=T_{0}-T_{1}>0$. The lateral boundaries are both vertical, located a distance $L$ from each other, and assumed to be impenetrable and exhibit zero-stress and zero heat-flux. The problem is simplified using the Boussinesq approximation, in which the density $\rho$ is considered as constant everywhere except for a vertical buoyancy force in which the density is assumed to depend linearly on temperature, as $\rho=\rho_{0}\left[1-\alpha\left(T-T_{0}\right)\right]$, where $\rho_{0}$ is the density at temperature $T_{0}$ and $\alpha$ is the thermal expansion coefficient. In a Cartesian coordinate system, the horizontal and vertical coordinates $x$ and $z$, respectively, the time $t$, the velocity $\boldsymbol{v}$, the pressure $P$, and the temperature $T$ are nondimensionalized as $x^{\prime}=x / d, z^{\prime}=z / d, t^{\prime}=\kappa t / d^{2}$, $\boldsymbol{v}^{\prime}=d \boldsymbol{v} / \kappa, P^{\prime}=d^{2} P /\left(\rho_{0} \kappa v\right)$, and $\theta^{\prime}=\left(T-T_{0}\right) / \Delta T$, respectively, where primes denote nondimensional quantities. Here, $\kappa$ is the thermal diffusivity and $v$ the viscosity, both assumed constant. Dropping primes, the nondimensional continuity, momentum, and energy conservation equations,

$\nabla \cdot \boldsymbol{v}=0$

$\frac{1}{\operatorname{Pr}}\left(\partial_{t} \boldsymbol{v}+\boldsymbol{v} \cdot \nabla \boldsymbol{v}\right)=R \theta \boldsymbol{e}_{z}-\nabla P+\Delta \boldsymbol{v}$,

$\partial_{t} \theta+\boldsymbol{v} \cdot \nabla \theta=\Delta \theta$

are applied in the domain $\Omega: 0<x<\Gamma, 0<z<1$, where $\Gamma=L / d$ is the aspect ratio, and are subject to the boundary conditions

$\boldsymbol{\nu}=\mathbf{0}, \theta-1=0 \quad$ at $z=0, \quad \theta=\partial_{z} u=w=0 \quad$ at $z=1$,

$\partial_{x} \theta=\partial_{x} w=u=0$ at $x=0$ and at $x=\Gamma$.

Here, $\boldsymbol{e}_{z}$ is the upwards vertical unit vector, $u$ and $w$ are the horizontal and vertical velocity components, respectively, $P r=v / \kappa$ is the Prandtl number, and $R=d^{3} \alpha g \Delta T /(v \kappa)$ is the Rayleigh number, where $g$ is the acceleration of the gravity.

The problem (1)-(5) has a simple conductive solution $\boldsymbol{v}^{c}=\mathbf{0}, \theta^{c}=1-z$, and $P^{c}=R\left(z-z^{2} / 2\right)$. Considering the limit $\operatorname{Pr} \rightarrow \infty$, as in [33], the left hand side term in Eq. (2) can be set equal to zero. Rescaling the problem as $\theta^{\prime}=\theta-\theta^{c}, P^{\prime}=\left(P-P^{c}\right) / R$, $v^{\prime}=v / \sqrt{R}, t^{\prime}=t \sqrt{R}$, neglecting the term of order $O(1 / P r)$ in the momentum equation, and dropping primes, the formulation (1)(3) is rewritten as

$\nabla \cdot \boldsymbol{v}=0$ in $\Omega$

$\theta \boldsymbol{e}_{z}-\nabla P+\frac{1}{\sqrt{R}} \Delta \boldsymbol{\nu}=0 \quad$ in $\Omega$,

$\partial_{t} \theta+\boldsymbol{v} \cdot \nabla \theta-w=\frac{1}{\sqrt{R}} \Delta \theta \quad$ in $\Omega$,

and boundary conditions (4) and (5) as,

$\boldsymbol{v}=\mathbf{0}, \theta=0 \quad$ at $z=0, \quad \theta=\partial_{z} u=w=0 \quad$ at $z=1$,

$\partial_{x} \theta=\partial_{x} w=u=0 \quad$ at $x=0$ and at $x=\Gamma$.

It is to be noted that with this scaling the conductive state is

$\boldsymbol{v}=\mathbf{0}, \quad \theta=0$.

Also, $|\boldsymbol{v}| \sim|\theta| \sim 1$, which means that neglecting the effect of the Prandtl number is justified provided that $\operatorname{Pr} \gg 1$, as readily seen for both $R \sim 1$ and $R \gg 1$ comparing the order of magnitudes of the neglected convective terms and the retained viscous terms, both in the bulk fluid and in the boundary layer attached to the lower plate that appears as $R \gg 1$ and exhibits a $O\left(R^{-1 / 4}\right)$-thickness.

The governing equations and boundary conditions (6)-(10) are invariant under the horizontal reflection symmetry

$\gamma:(\chi, z, u, w, \theta, P) \rightarrow(-\chi, z,-u, w, \theta, P)$.
On the other hand, if Eqs. (6)-(9) are considered in an infinite slab and the solutions are required to be both $2 \Gamma$-periodic in the $x$ direction and reflection symmetric, then the restriction of this problem to a half of the period precisely obeys (10). Restricting to the fixed domain eliminates invariance under arbitrary translations of the problem in the infinite domain, but invariance under certain translations will still be appreciated in some specific patterns; see below.

Eqs. (6)-(10) are discretized using a Legendre spectral collocation method [9,11], retaining polynomials of degrees $n=17$ and $m=13$ in the $x$ and $z$ directions, respectively, with the $I=18 \times 14=252$ collocation points symmetrically located in both the $x$ and $z$ directions. Thus, the reflection symmetry (12) is easily identified in the values of $\boldsymbol{v}, P$, and $\theta$ at the collocation points. As reported in [33], this discretization is fine enough to describe the bifurcation diagram considered below. It must be noted, however, that the ROMs developed below are somehow transparent to the number of collocation points because (i) the results provided by the ROMs will be compared with their counterparts in the Legendre solver; and (ii) the outcomes of the ROMs will be given as linear combinations of the POD modes, which in turn will be calculated precisely at Legendre collocation points. This strategy avoids interpolation between different grids, which would add spurious redundancies that would mask the true redundancies identified in the snapshots by POD. Using the Legendre collocation points, the integrals in the domain (for, e.g., the temperature field $\theta$ ) are approximated as

$\int_{\Omega} \theta(x, z) d x d z \simeq \sum_{i=1}^{I} g_{i} \theta_{i}$

where $i$ indicates the various collocation points in the grid and $g_{i}$ are the associated Legendre-Gauss-Lobato (LGL) weights. Using this, the usual $L^{2}$-inner products of vector and scalar functions are approximated as

$$
\begin{aligned}
\left\langle\theta^{1}, \theta^{2}\right\rangle_{1}= & \int_{\Omega} \theta^{1} \theta^{2} d x d z \simeq \theta^{1 \top} \mathcal{G} \theta^{2} \\
\left\langle\boldsymbol{v}^{1}, \boldsymbol{v}^{2}\right\rangle_{2}= & \int_{\Omega} \boldsymbol{v}^{1} \cdot \boldsymbol{v}^{2} d x d z=\left\langle u^{1}, u^{2}\right\rangle_{1}+\left\langle w^{1}, w^{2}\right\rangle_{1} \simeq \boldsymbol{u}^{1 \top} \mathcal{G} \boldsymbol{u}^{2} \\
& +\boldsymbol{w}^{1 \top} \mathcal{G} \boldsymbol{w}^{2}
\end{aligned}
$$

where $\boldsymbol{v}^{i}=\left(\boldsymbol{u}^{i}, w^{i}\right), \boldsymbol{i}=1,2$, and the vectors $\boldsymbol{\theta}^{1,2}, \boldsymbol{u}^{1,2}$, and $\boldsymbol{w}^{1,2}$ contain the values of $\theta^{1,2}, u^{1,2}$, and $w^{1,2}$ at the collocation points and $\mathcal{G}$ is the diagonal matrix whose elements are the LGL weights. For the

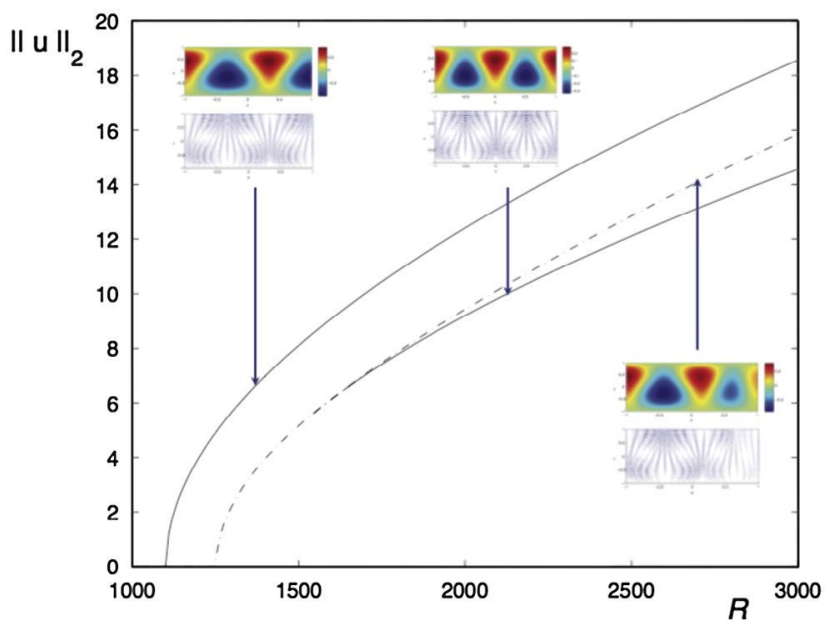

Fig. 2. Bifurcation diagram of the problem (6)-(10) at $\Gamma=3.495$, plotting the $L^{2}$ norm $\mid u \|_{2}$ vs. the Rayleigh number $R$. Stable branches are solid while unstable are dashed with dots. 
sake of clarity, caligraphic boldface will be used hereafter to denote matrices, while vectors containing the values of scalar variables at the collocation points will be denoted using roman boldface. To avoid confusion, the velocity components $u$ and $w$ will be used hereafter, instead of the velocity vector $\boldsymbol{v}$.

In order to facilitate comparison with [33], the specific value $\Gamma=3.495$ is considered for the aspect ratio in the applications below, where the Rayleigh number $R$ will be varied in the range $0 \leqslant R \leq 3000$. A linear stability analysis of the steady states of (6)-(10) has been performed in [33] considering the linearized eigenvalue problem

$\nabla \cdot \widetilde{\boldsymbol{v}}=0$ in $\Omega$,

$\widetilde{\theta} \boldsymbol{e}_{z}-\nabla \widetilde{P}+\frac{1}{\sqrt{R}} \Delta \widetilde{\boldsymbol{v}}=0 \quad$ in $\Omega$,

$-\widetilde{\boldsymbol{v}} \cdot \nabla \theta-\boldsymbol{v} \cdot \nabla \tilde{\theta}+\widetilde{\boldsymbol{w}}+\frac{1}{\sqrt{R}} \Delta \widetilde{\theta}=\lambda \widetilde{\theta} \quad$ in $\Omega$,

with boundary conditions,

$\widetilde{\boldsymbol{v}}=\mathbf{0}, \widetilde{\theta}=0 \quad$ at $z=0, \quad \widetilde{\theta}=\partial_{z} \widetilde{u}=\widetilde{\mathcal{W}}=0 \quad$ at $z=1$,

$\partial_{x} \widetilde{\theta}=\partial_{x} \widetilde{w}=\widetilde{u}=0 \quad$ at $x=0$ and at $x=\Gamma$,

which is obtained perturbing $(\boldsymbol{v}, P, \theta)$ as $(\boldsymbol{v}, P, \theta)+(\widetilde{\boldsymbol{v}}, \widetilde{P}, \widetilde{\theta}) \mathrm{e}^{\lambda t}$ in $(6)-(10)$ and linearizing. For the conductive state (11), the left hand sides of (16)-(18) define a linear operator that is self-adjoint with the $L^{2}$-inner product (thus, all eigenvalues are real in this case).

The bifurcation diagram giving the steady states for varying $R$ is given in Fig. 2, as calculated in [33], plotting the $L^{2}$-norm of the horizontal velocity component,

$\|\boldsymbol{u}\|_{2}=\sqrt{\int_{-1}^{1} \int_{-1}^{1}|\boldsymbol{u}(x, z)|^{2} d x d z}$

vs. $R$. As can be seen, the conductive branch (11) exhibits two bifurcations in this range of $R$. A first symmetry breaking (namely, pitchfork [27,22]) supercritical bifurcation occurs at $R=R_{c}^{1}=1100$, where the symmetry (12) is broken. A second pitchfork bifurcation at $R=R_{c}^{2}=1252$ produces two different branches of reflection symmetric solutions (namely, invariant under (12)). The bifurcation is pitchfork (and not transcritical) because it breaks the symmetry $x \rightarrow x+0.5$, which is a symmetry of the extension of the problem to the infinite slab, as explained above. The symmetry (12) of the bifurcated branches is broken at two (one per branch) subcritical pitchfork bifurcations that occur at $R=R_{c}^{3}=1538$ in both branches and produce four unstable bifurcated branches; the branches of horizontally symmetric solutions gain stability at these points, as indicated in Fig. 2.

Following ideas from [50], the intention in this paper is to calculate this bifurcation diagram using a POD + Galerkin projection ROM based on a fairly computationally inexpensive preprocess. The ROM will either preserve the exact horizontal reflection symmetry or not, comparing the outcomes of these strategies. The POD modes are calculated from snapshots computed in two ways, either from snapshots calculated by a time-dependent solver on (6)-(10) or as iterates of a Newton method, both based on Legendre collocation. But the application of these ideas requires an efficient calculation of the POD modes themselves, which is considered in the next section and is crucial to extract enough information from the snapshots, as explained below. In addition, combining POD with the symmetries of the problem is convenient.

\section{Efficient calculation of POD modes}

POD modes (and singular values) will be calculated independently for the velocity/pressure and temperature fields, and will be called hydrodynamic and thermal modes, respectively. For illustration, two sets of snapshots are calculated integrating Eqs. (6)(10) with the Legendre CFD solver and the following initial condition

$(\boldsymbol{v}, P, \theta)=\alpha_{1}\left(\widetilde{\boldsymbol{v}}_{1}, \widetilde{P}_{1}, \widetilde{\theta}_{1}\right)+\alpha_{2}\left(\widetilde{\boldsymbol{v}}_{2}, \widetilde{P}_{2}, \widetilde{\theta}_{2}\right), \quad$ at $t=0$,

where $\alpha_{1}=\alpha_{2}=0.1$, and $\left(\widetilde{\boldsymbol{v}}_{1}, \widetilde{P}_{1}, \widetilde{\theta}_{1}\right)$ and $\left(\widetilde{\boldsymbol{v}}_{2}, \widetilde{P}_{2}, \widetilde{\theta}_{2}\right)$ are unit eigenvectors of (16)-(20) associated with the two most unstable eigenvalues at:

- The conductive state at $R=1000$.

- The upper bifurcated state (see Fig. 2) at $R=1500$. Note that the conductive state cannot be considered at $R=1500$ because this state is unstable.

This gives, in both cases, $\theta, u, w$, and $P$ as functions of $(x, z, t)$. The thermal and hydrodynamic snapshots are defined as the uncoverged states

$\theta_{j}=\theta\left(x, z, t_{j}\right), \quad\left(u_{j}, w_{j}, P_{j}\right)=(u, w, P)\left(x, z, t_{j}\right)$,

where $t=j \delta$, with $\delta=0.01$ in both cases. The numbers of snapshots at $R=1000$ and 1500 are 30 and 40 , respectively, meaning that the time span in which the snapshots are calculated in these two cases are $0 \leq t \leq 30 \times 0.01=0.3$ and $0 \leq t \leq 0.4$, respectively. Both, the time shift $\delta$ and the numbers of snapshots have been chosen after some calibration.

As is well-known [20], the standard method (based on the covariance matrix) to calculate POD modes and singular values is strongly affected by round off errors, which essentially halve the precision of the calculations (namely, provide results within $10^{-8}$ accuracy performing calculations in double precision). The difficulty is usually overcome using a more convenient method that, for the standard Euclidean product, is implemented in the Matlab routine 'svd'. This routine will be combined in Section 3.2 with some additional ingredients.

\subsection{The standard method}

For the sake of clarity, let us begin with the thermal modes, considering the thermal snapshots matrix, $\mathcal{S}$, whose columns $\left(\boldsymbol{S}_{1}, \boldsymbol{S}_{2}, \ldots\right)$ are the thermal snapshots, defined in (23). The standard method to calculate POD modes proceeds in three steps. First, (i) the covariance matrix is defined in terms of the snapshots as

$\mathcal{R}=\mathcal{S}^{\top} \mathcal{G S}$

Namely, the elements of this (positive, semi-definite) matrix are the products of the snapshots with the inner product defined in (14), namely $\mathcal{L}_{i j}=\left\langle\boldsymbol{S}_{i}, \boldsymbol{S}_{j}\right\rangle_{1}$. Then, (ii) the eigenvalues and eigenvectors of $\mathcal{R}$ are calculated, which gives

$\mathcal{R} \mathcal{V}=\mathcal{V D}^{2}$

where the columns of the (generally rectangular) matrix $\mathcal{V}$ are orthonormal eigenvectors associated with strictly positive eigenvalues, meaning that $\mathcal{V}$ is unitary, namely

$\mathcal{V}^{\top} \mathcal{V}=\mathcal{I}=$ unit matrix

and $\mathcal{D}^{2}$ is the diagonal matrix whose elements are the strictly positive eigenvalues, whose square roots are the POD singular values. As a third step, (iii) the POD modes associated with strictly positive singular values, $\mathbf{Q}^{1}, \ldots, \mathbf{Q}^{\prime}$, are the columns of the matrix $\mathcal{Q}$, which is given by

$\mathcal{Q}=\mathcal{S} \mathcal{V D}^{-1}$.

The POD modes are orthonormal with the inner product (14) because 


$$
\begin{aligned}
\mathcal{Q}^{\top} \mathcal{G Q} & =\mathcal{D}^{-1} \mathcal{V}^{\top}\left(\mathcal{S}^{\top} \mathcal{G S}\right) \mathcal{V} \mathcal{D}^{-1}=\mathcal{D}^{-1} \mathcal{V}^{\top}(\mathcal{R} \mathcal{V}) \mathcal{D}^{-1} \\
& =\mathcal{D}^{-1} \mathcal{V}^{\top} \mathcal{V} \mathcal{D}^{2} \mathcal{D}^{-1}=\mathcal{I}
\end{aligned}
$$

as subsequently obtained using (24)-(27). This identity holds only if all operations are exact. But, because of the division by very small singular values in (27), the columns of $\mathcal{Q}$ are slightly non-orthonormal. These can be re-ortonormalized using a Gramm-Schmidt method based on the inner product (14).

For the sake of clarity, the algorithm to calculate the thermal modes is summarized below; hydrodynamic modes are calculated similarly.

\section{Algorithm 1. Standard method.}

Step 1 Construct the thermal snapshots matrix $\mathcal{S}^{\theta}=\left[\theta_{1} \theta_{2} \ldots\right.$ $\left.\theta_{N}\right] \in \mathcal{M}_{1 \times N}$, whose columns are the values of the thermal snapshots defined after $(23)$ in the $I=(n+1) \times$ $(m+1)$ collocation points.

Step 2 Obtain the covariance matrix, $\mathcal{R}^{\theta}=\left(\boldsymbol{S}^{\theta}\right)^{T} \mathcal{G S}^{\theta} \in$ $\mathcal{M}_{N \times N}$, where $\mathcal{G} \in \mathcal{M}_{I \times I}$ is the diagonal matrix whose elements are the LGL weights.

Step 3 Calculate the spectral decomposition of the positive semi-definite matrix $\mathcal{R}^{\theta}$ using the Matlab command eigs, as $\mathcal{R}^{\theta}=\mathcal{V}^{\theta}\left(\mathcal{D}^{\theta}\right)^{2}\left(\mathcal{V}^{\theta}\right)^{T}$, defined such that eigenvalues of $\mathcal{R}^{\theta}$ are the diagonal elements of $\left(\mathcal{D}^{\theta}\right)^{2}$ and the eigenvectors are the columns of $\mathcal{V}^{\theta}$.

Step 4 Calculate the POD thermal modes associated with the $J$ unsaturated singular values as $\mathcal{Q}_{\theta}^{j}=\sum_{i=1}^{N} \mathcal{V}_{i j}^{\theta} \theta_{i}$, $j=1,2, \ldots, J$.

Step 5 Re-orthonormalize the basis $\mathcal{B}_{\theta}^{\text {POD }}=\left\{\mathcal{Q}_{\theta}^{1}, \ldots, \mathcal{Q}_{\theta}^{\prime}\right\}$ by applying a Gramm-Schmidt method with the inner product in $L^{2}$ defined in (14).

Because of round off errors resulting from the multiplication of snapshots in (24), this standard method calculates the singular values $\sigma_{j}$ and the POD modes $\mathbf{Q}^{j}$ up to round off errors that (performing calculations with double precision) scale with

error $_{j} \sim \sigma_{1}^{2} 10^{-16} / \sigma_{j}^{2}$.

This error estimate is tested for the two sets of snapshots defined after Eq. (22) in Fig. 3, where errors are calculated considering as exact the POD modes provided by the modified method described in the Section 3.2 below and the estimate is based on the singular values, plotted with crosses in Fig. 4 below. Note that the errors conform reasonably well the estimate (30). Also note that for $R=1000$, only 6 POD modes are useful; the remaining 24 POD modes exhibit $O(1)$ errors and are useless; similarly, only 12 modes are useful for $R=1500$. Using the estimate (29), the number $J$ of retained POD modes for a prescribed accuracy $\varepsilon$ can be chosen as

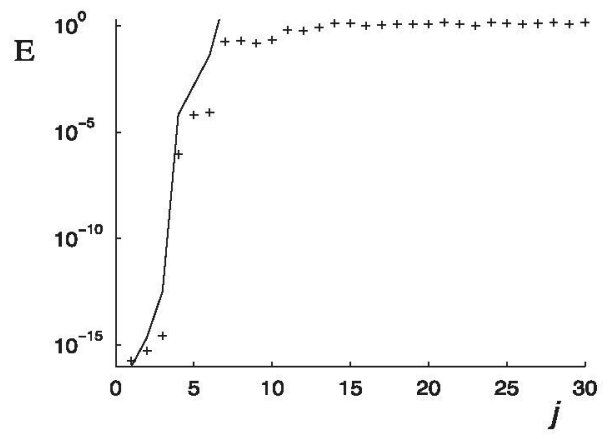

$\sigma_{1}^{2} 10^{-16} / \sigma_{J}^{2} \geqslant \varepsilon$

The strong loss of accuracy (the double precision accuracy is halved!) in this standard method is not even mentioned in papers dealing with POD-based reduced order modeling, perhaps because no intention is usually made to retain POD modes associated with such small singular values. In this paper, instead, as in [36,49-51], we shall retain as many POD modes as possible.

\subsection{The modified method}

As anticipated, the reason for the inability of the standard method to calculate POD modes associated with very small singular values is related to round off errors in the calculation of the covariance matrix (24). The problematic product of snapshots in (24) can be avoided applying SVD, as has been repeatedly done in various papers $[36,49-51]$. The modified method is briefly described here using the inner product (14), as follows. First, apply SVD (using the quite efficient MatLab command svd-econ) to the matrix

$\widetilde{\mathcal{S}}=\sqrt{\mathcal{G} \mathcal{S}}$

which is the result of multiplying in the snapshots matrix the values of the temperature at the collocation points by the square roots of the associated LGL weights. It follows that

$\widetilde{\mathcal{S}}=\mathcal{U} \mathcal{D} \mathcal{V}^{\top}$

The SVD decomposition (32) provides, retaining only the strictly positive singular values, the diagonal matrix $\mathcal{D}$ and the unitary matrix $\mathcal{V}$ that are readily seen to satisfy (25), which is seen (i) noticing that since

$\mathcal{S}=(\sqrt{\mathcal{G}})^{-1} \widetilde{\mathcal{S}}=(\sqrt{\mathcal{G}})^{-1} \mathcal{U} \mathcal{D} \mathcal{V}^{\top}$

and that (ii) the covariance matrix can be written as

$\mathcal{R}=\mathcal{S}^{\top} \mathcal{G S}=\mathcal{V} \mathcal{D} \mathcal{U}^{\top}(\sqrt{\mathcal{G}})^{-1} \mathcal{G}(\sqrt{\mathcal{G}})^{-1} \mathcal{U} \mathcal{D} \mathcal{V}^{\top}=\mathcal{V} \mathcal{D}^{2} \mathcal{V}^{\top}$

where it has been taken into account that $\mathcal{D}$ and $\mathcal{G}$ are both diagonal and $\mathcal{U}$ is unitary, then it is proved that $\mathcal{D}$ and $\mathcal{V}$ satisfy (25), as stated. POD modes are calculated substituting these matrices into (27). As in the standard method, because of division by $\sigma_{j}$, the POD modes calculated via (27) are slightly non-orthonormal. Again, they can be reorthonormalized by applying the Gramm-Schmidt method with the inner product (14), which yields (cf. (27))

$\mathcal{Q}=\mathcal{S} \mathcal{A}, \quad$ with $\mathcal{A}=\mathcal{V} \mathcal{D}^{-1} \mathcal{T}$

where $\mathcal{T}$ is a lower triangular, near-identity matrix resulting from Gramm-Schmidt.

As in the previous subsection, the algorithm is summarized here for the thermal modes. Again, the hydrodynamic modes are treated similarly.

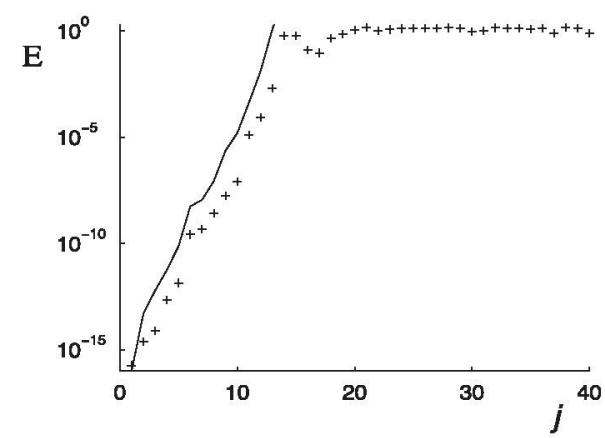

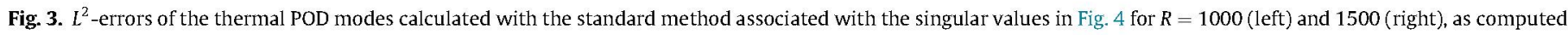
comparing with the more exact method described in Section 3.2 (crosses); the estimate (29) is plotted with solid line. 

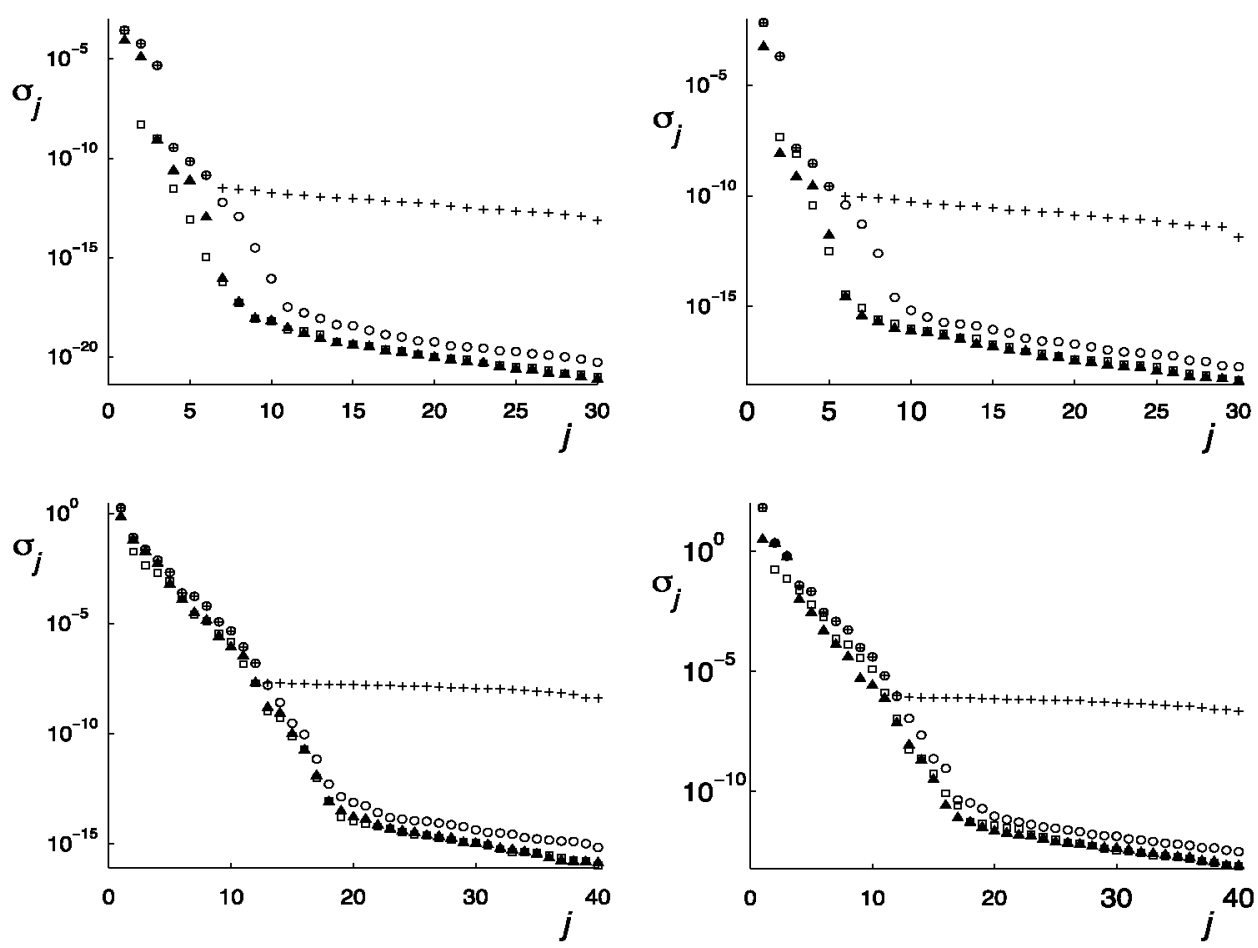

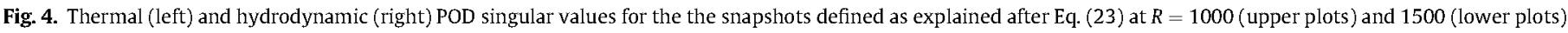

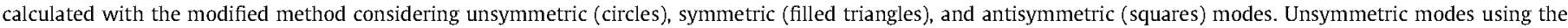
standard method are plotted with crosses for reference.

Algorithm 2. Modified method.

Step 1 Construct the modified thermal snapshots matrix $\widetilde{\mathcal{S}}^{\theta}=\sqrt{\mathcal{G}} \mathcal{S}^{\theta}$, where $\mathcal{G}$ is the diagonal matrix whose diagonal terms are the LGL weights and $\mathcal{S}^{\theta}=$ $\left[\theta_{1} \theta_{2} \ldots \theta_{N}\right]$ is the ordinary snapshots matrix, whose columns are the values of the thermal snapshots defined after (23).

Step 2 Calculate the singular value decomposition of $\widetilde{\mathcal{S}}^{\theta}$ using the Matlab command svd-'econ', as $\tilde{\mathcal{S}}^{\theta}=$ $\mathcal{U}^{\theta} \mathcal{D}^{\theta}\left(\mathcal{V}^{\theta}\right)^{T}$

Step 3 Calculate the POD thermal modes asociated with the $J$ unsaturated singular values as $\mathcal{Q}_{\theta}^{j}=\sum_{i=1}^{N} \mathcal{V}_{i j}^{\theta} \theta_{i}$, $j=1,2, \ldots, J$.

Step 4 Re-orthonormalize the basis $\mathcal{B}_{\theta}^{\text {POD }}=\left\{\mathcal{Q}_{\theta}^{1}, \ldots, \mathcal{Q}_{\theta}^{J}\right\}$ by applying a Gramm-Schmidt method with the inner product in $L^{2}$ defined in (14).

This method provides the singular values and modes with a precision that scales as

error $_{j} \sim \sigma_{1} 10^{-16} / \sigma_{j}$,

meaning that the number of retained modes for a given accuracy is given by

$\sigma_{1} 10^{-16} / \sigma_{J} \leqslant \varepsilon$

Comparison with (29) and (30) shows that the new method essentially (i) doubles the precision of the calculation of POD modes and singular values and (ii) is able to extract more POD modes than the standard method from the same set of snapshots. This is quite important since it allows from maximizing the information extracted from a given system of snapshots, whose calculation is usually the most computationally expensive part of the process.
For illustration, the modified method is applied to the thermal snapshots defined after Eq. (23) and the resulting singular values plotted in Fig. 4-left, where the singular values provided by the standard method are also plotted for reference. Note that, as anticipated, the modified method essentially doubles the precision of the standard method for higher order modes. Thus, it will be this more precise POD method that will be used in the remaining of the paper. At the moment, note that imposing the accuracy $\varepsilon=10^{-3}$ in (37), the method selects 9 and 15 POD thermal modes at $R=1000$ and 1500 , respectively, and that, according to (36), these modes are calculated with a very good precision. The estimate (30) for the standard method, instead, only provides 5 and 10 thermal modes, respectively, with the remaining modes being essentially useless. Also note that the number of useful modes is much larger when the snapshots result from the perturbed dynamics of the bifurcated state at $R=1500$ than when they result from perturbations of the conductive state, at $R=1000$. This is because the dynamics near the non-conductive state is richer.

The 12 most energetic thermal POD modes associated with the singular values at $R=1000$ are plotted in the first two rows of Fig. 5. As expected, the spatial complexity of the modes increases as singular values decreases. Note that some modes (e.g., the third mode) are approximately symmetric and some others (e.g., the first mode), approximately antisymmetric, but the remaining modes (e.g., the second mode) do not show any symmetry, even qualitatively.

The partial derivatives of the thermal POD modes are readily calculated invoking (38) and noting that the partial derivatives are associated with linear operators. In other words, the partial derivatives of the modes are obtained by post-multiplying by the matrix $\mathcal{A}$ the matrices that give the corresponding partial derivatives of the snapshots. In particular, the first order partial derivatives, $\partial_{x}$ and $\partial_{y}$, and the Laplacian (appearing in (8)) of the thermal modes are given by 


\section{UNSYMMETRIC MODES}
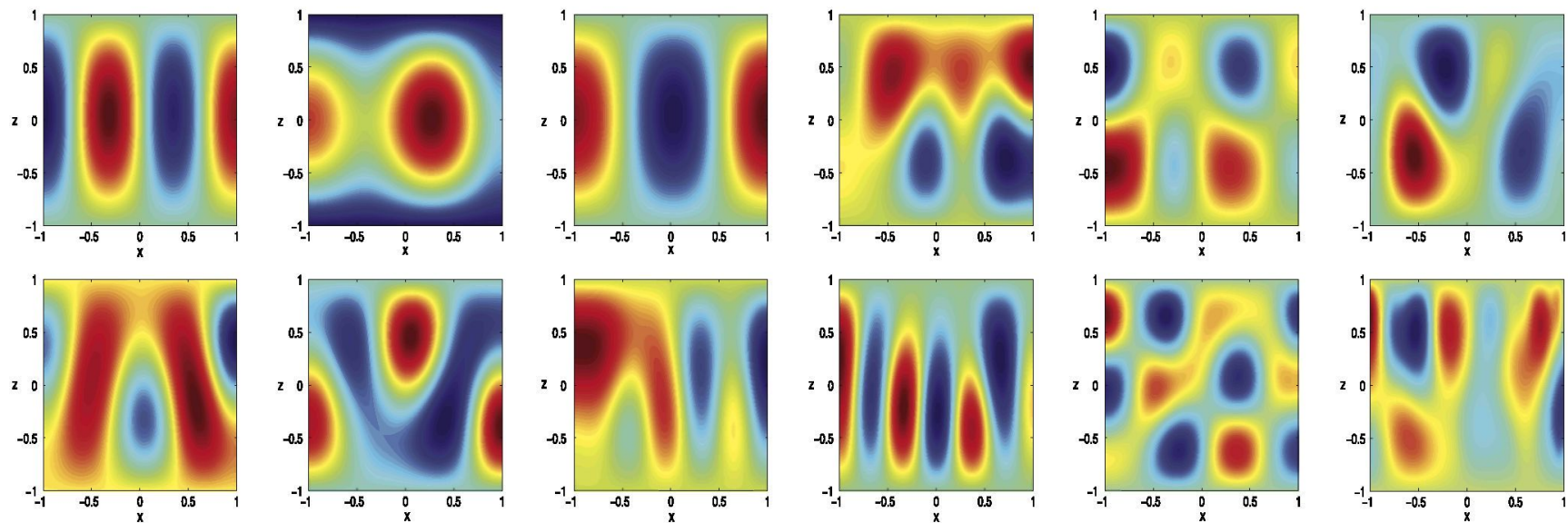

\section{HORIZONTALLY SYMMETRIC/ANTISYMMETRIC MODES}
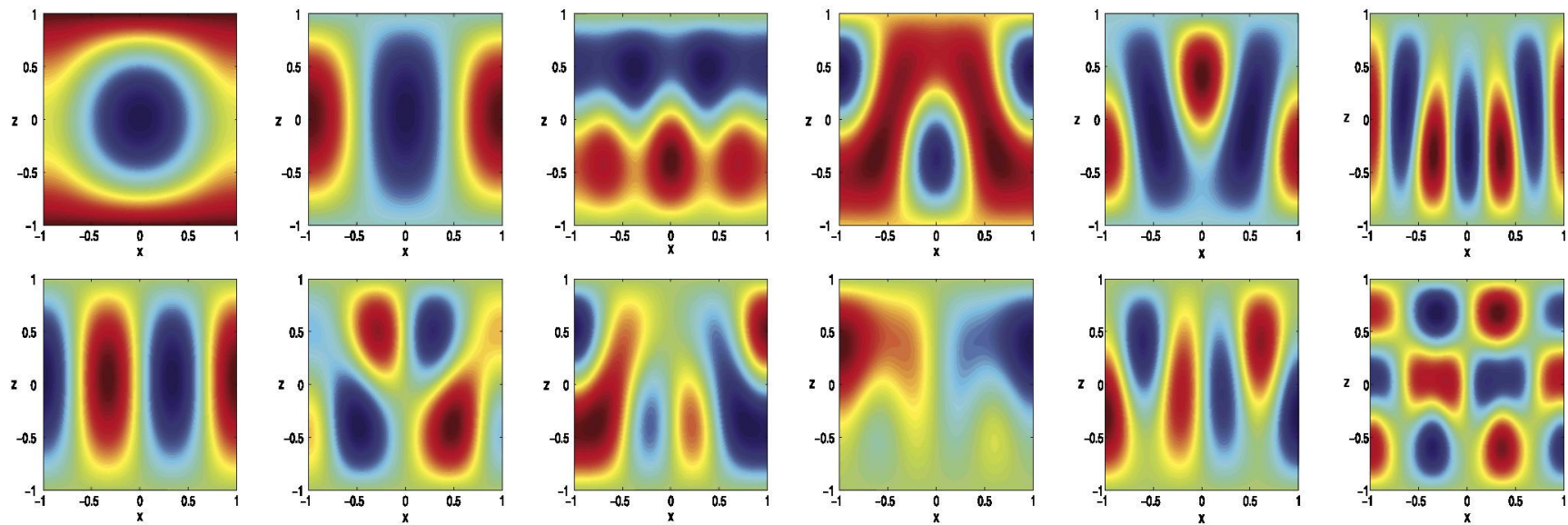

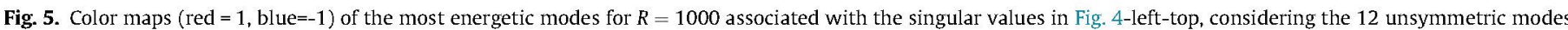

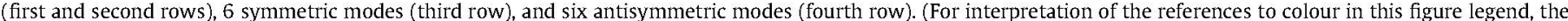
reader is referred to the web version of this article.)

$\mathcal{L}^{\partial_{x}} \mathcal{Q}=\left(\mathcal{L}^{\partial_{x}} \mathcal{S}\right) \mathcal{A}, \quad \mathcal{L}^{\partial_{y}} \mathcal{Q}=\left(\mathcal{L}^{\partial_{y}} \mathcal{S}\right) \mathcal{A}, \quad \mathcal{L}^{\Delta} \mathcal{Q}=\left(\mathcal{L}^{\Delta} \mathcal{S}\right) \mathcal{A}$

respectively. Here, $\mathcal{L}^{\partial_{x}}, \mathcal{L}^{\partial_{z}}$, and $\mathcal{L}^{\Delta}$ are the Legendre collocation matrices associated with the operators $\partial_{x}, \partial_{z}$, and $\Delta$, respectively.

Hydrodynamic POD modes, namely modes associated with the velocity/pressure components, are calculated similarly, considering joint snapshots (according to (23)) and joint modes for the two velocity components and the pressure, and using the inner product (15). In other words, the hydrodynamic snapshots matrix, whose columns are the values the hydrodynamic variables at the $I$ collocation points, namely

$\left[u_{1}, \ldots, u_{I}, w_{1}, \ldots, w_{I}, P_{1}, \ldots, P_{I}\right]^{\top}$

is weighted with the LGL weights to obtain the weighted snapshots matrix, $\widetilde{\mathcal{S}}$, whose columns are (cf. (31))

$\left(\sqrt{g_{1}} u_{1}, \ldots, \sqrt{g_{I}} u_{I}, \sqrt{g_{1}} w_{1}, \ldots, \sqrt{g_{I}} w_{I}, \sqrt{g_{1}} P_{1}, \ldots, \sqrt{g_{I}} P_{I}\right)^{\top}$.

Applying SVD to the weighted snapshots matrix $\widetilde{\mathcal{S}}$, and retaining only the strictly positive singular values yields the decomposition (32), provides the singular values in the diagonal matrix $\mathcal{D}$ and the unitary matrix $\mathcal{V}$. The counterpart of (35) provides the matrix $\mathcal{A}$ and the velocity and pressure components of the hydrodynamic modes, $\mathcal{Q}_{u}, \mathcal{Q}_{w}$, and $\mathcal{Q}_{P}$, as
$\mathcal{Q}=\left[\begin{array}{l}\mathcal{Q}_{u} \\ \mathcal{Q}_{w} \\ \mathcal{Q}_{p}\end{array}\right]=\mathcal{S A}$

where the joint snapshots matrix $\mathcal{S}$ is defined such that its columns are the joint snapshots (39). Note that the pressure has not been considered in the calculation of the velocity modes and singular values, but it has been incorporated into the play only in Eq. (41) by imposing that the pressure components of the hydrodynamic modes are given by the same linear combination (namely, same matrix $\mathcal{A}$ in (41)) of snapshots as the velocity components. This means that the pressure is made somehow slaved to the velocity in this calculation. This is consistent with the standard way of treating the pressure in incompressible fluid mechanics, where the pressure can be seen as a Lagrange multiplier when the continuity equation is considered as a restriction to the momentum equations.

As it happens with thermal modes, the resulting hydrodynamic modes may be slightly oblique with the inner product (15) due to round off errors. Re-orthonormalizing these POD modes with the Gramm-Schmidt method using the inner product (15) slightly modifies the matrix $\mathcal{A}$ in (41). As in thermal modes, this matrix can also be used to calculate the partial derivatives of the POD modes in terms of their counterparts for the pressure. In particular, 
the components of the gradient of pressure and the Laplacian of the velocity, appearing in (38) are calculated using the counterpart of Eq. (38), which reads

$$
\begin{gathered}
\mathcal{L}^{\partial_{x}} \mathcal{Q}_{P}=\left(\mathcal{L}^{\partial_{x}} \mathcal{S}_{P}\right) \mathcal{A}, \quad \mathcal{L}^{\partial_{y}} \mathcal{Q}_{P}=\left(\mathcal{L}^{\partial_{y}} \mathcal{S}_{P}\right) \mathcal{A}, \\
\mathcal{L}^{\Delta} \mathcal{Q}_{u}=\left(\mathcal{L}^{\Delta} \mathcal{S}_{u}\right) \mathcal{A}, \quad \mathcal{L}^{\Delta} \mathcal{Q}_{w}=\left(\mathcal{L}^{\Delta} \mathcal{S}_{w}\right) \mathcal{A}
\end{gathered}
$$

where the subscripts $P, u$, and $w$ in the snapshots matrix indicate those blocks of $\mathcal{S}$ associated with the pressure and the velocity components. The hydrodynamic singular values resulting from the hydrodynamic snapshots calculated as explained above are plotted in Fig, 4-right, both as calculated by this modified method and by the standard method. Note that the hydrodynamic modes follow similar trends as the thermal modes discussed above, which means that thermal and hydrodynamic modes are strongly correlated. This could suggest considering joint hydrodynamic/thermal modes in the Galerkin projection, which could be appropriate to construct some particular solutions. Nevertheless, the velocity and temperature fields play a fairly different role in the conductive and convective solutions, and thus independent hydrodynamic and thermal modes will be used in the remaining of the paper.

\subsection{Reflection symmetric and anti-symmetric POD modes}

As calculated above, hydrodynamic and thermal POD modes have been calculated ignoring the exact reflection symmetry (12). As a consequence, the Galerkin system obtained in the next section using these modes will not preserve this symmetry. On the other hand, because of invariance of the governing equations under the symmetry (12), the result of applying this symmetry to the POD modes are also acceptable POD modes, which in principle (except for redundancies among the resulting set of modes) doubles the number of POD modes that are extracted from a given set of snapshots. This is quite convenient since computation of snapshots is the most computationally expensive part of the process. Both aims, namely obtaining a symmetry preserving Galerkin system (in Section 4 below) and enlarging the number of useful POD modes are reached considering reflection symmetric and anti-symmetric modes that, as in [42,43], are calculated in two steps. Namely, (i) the snapshots are decomposed into their reflection symmetric and anti-symmetric parts, as

$(u, w, P, \theta)^{ \pm}(\chi, z)=[(u, w, P, \theta)(\chi, z) \pm(-u, w, P, \theta)(-\chi, z)] / 2$,

and (ii) POD is applied separately (as explained in Section 3.2) to the hydrodynamic and thermal parts of the symmetric/antisymmetric snapshots, which produces symmetric and antisymmetric singular values and POD modes, denoted hereafter with the superscripts + and -, respectively. Note that now, the error estimate and the selection of the number of modes to be retained in the symmetric and antisymmetric modes, $J^{+}$and $J^{-}$, respectively, (36) and (37) must be replaced by

$\operatorname{error}_{j}^{+} \sim \max \left\{\sigma_{1}^{+}, \sigma_{1}^{-}\right\} 10^{-16} / \sigma_{j}^{+}$,

meaning that the number of retained modes for a given accuracy is given by

$\max \left\{\sigma_{1}^{+}, \sigma_{1}^{-}\right\} 10^{-16} / \sigma_{j^{ \pm}}^{ \pm} \leqslant \varepsilon$

For illustration, the reflection-symmetric and anti-symmetric, hydrodynamic and thermal singular values calculated from the snapshots (23) are plotted in Fig. 4, where comparison is visible with the singular values that result ignoring the symmetry. Note that taking the required accuracy $\varepsilon=10^{-3}$, Eq. (45) selects $J_{\theta}=J_{\theta}^{+}+J_{\theta}^{-}=5+5=10$ and $14+14=28$ thermal modes for $R=1000$ and 1500 , respectively, while the counterparts of these numbers were 9 and 15 , respectively, when the symmetry was ignored. Thus, the effect of the symmetry is quite clearly appreciated at $R=1500$, where the number of retained modes is essentially doubled by the symmetry, which is due to the fact that the dynamics implicit in the snapshots is richer in this case. Fig. 4 also shows that the hydrodynamic modes follow a similar trend. Thus, taking the exact symmetry into account increases the number of useful POD modes, as anticipated.

In order to further appreciate the role of the horizontal symmetry, the six most energetic symmetric and antisymmetric thermal POD modes associated with the singular values in Fig. 4 are provided in the third and fourth rows in Fig. 5, where comparison with the unsymmetric modes in the first two rows leads to two interesting conclusions. On the one hand, the second, fourth, fifth, and sixth symmetric modes and the first antisymmetric modes are fairly close to some unsymmetric modes, but these are calculated now more accurately; for instance, the sixth symmetric mode satisfies (44) with $\varepsilon=10^{-5}$, while the 11 th unsymmetric mode only satisfies (37) with $\varepsilon=10^{-1}$. In contrast, some other symmetric/antisymmetric modes (e.g., the first symmetric mode) show a new structure, not apparent in the unsymmetric modes, which illustrates the ability of the symmetric POD method to uncover more patterns than the standard POD in the set of snapshots. Summarizing, Fig. 5 confirms that, as anticipated, the symmetric POD method enlarges the number of useful POD modes that can be extracted from a given set of snapshots.

\section{A preprocessed $\mathrm{POD}+$ Galerkin projection method to calculate the bifurcation diagram}

Let us first construct the Galerkin system and then use it to calculate the bifurcation diagram for (6)-(10), associated with steady solutions. For the sake of clarity, the Galerkin system is first described in Section 4.1 projecting on the hydrodynamic and thermal unsymmetric POD modes calculated in Section 3.2. Then, a horizontal symmetry preserving Galerkin system is derived in Section 4.2 that takes the exact horizontal symmetry into account, using the POD modes calculated as explained in Section 3.3.

The Galerkin systems can be used to calculate bifurcation diagrams as explained in Section 4.3, using a simple continuation method. These methods are applied to the thermal convection problem in Section 5, where the snapshots will result from runs of either a time dependent solver (already used for illustration in Section 3) or a Newton method.

\subsection{The Galerkin system}

The steady solutions of (6)-(10) are sought expanding the velocity/pressure and temperature fields at the collocation points into POD modes as

$(\boldsymbol{u}, \boldsymbol{w}, \boldsymbol{P}) \simeq\left(\boldsymbol{u}^{\mathrm{GS}}, \boldsymbol{w}^{\mathrm{GS}}, \boldsymbol{P}^{\mathrm{GS}}\right)=\sum_{j=1}^{J_{v}} A_{j}\left(\boldsymbol{Q}_{u}^{j}, \mathbf{Q}_{w}^{j}, \boldsymbol{Q}_{P}^{j}\right), \quad \theta \simeq \theta^{\mathrm{GS}}=\sum_{j=1}^{J_{\theta}} B_{j} \mathbf{Q}_{\theta}^{j}$,

where $\left(\boldsymbol{Q}_{u}^{j}, \boldsymbol{Q}_{w}^{j}, \boldsymbol{Q}_{\mathrm{P}}^{j}\right)$ and $\boldsymbol{Q}_{\theta}^{j}$ are the hydrodynamic and thermal POD modes, computed as explained in Section 3.2; the numbers of retained hydrodynamic and thermal modes, $J_{\nu}$ and $J_{\theta}$ are selected as also explained in Section 3.2. The hydrodynamic and thermal amplitudes, $A_{j}$ and $B_{j}$, are calculated substituting (46) into (7) and (8) and multiplying the resulting equations by $\boldsymbol{Q}_{v}^{j}$ and $\boldsymbol{Q}_{\ominus}^{j}$ with the inner products (15) and (14), respectively, which yields the following Galerkin system (GS)

$\mathcal{L}^{11} \mathbf{A}+\mathcal{L}^{12} \mathbf{B}=0, \quad d \mathbf{A} / d t=\mathcal{L}^{21} \mathbf{A}+\mathcal{L}^{22} \mathbf{B}-\boldsymbol{\beta}(\mathbf{A}, \mathbf{B})$, 
where the $\mathbf{A}=\left(A_{1}, \ldots, A_{J_{v}}\right)^{\top}$ and $\mathbf{B}=\left(B_{1}, \ldots, B_{J_{\theta}}\right)^{\top}$ are the amplitude vectors, and the components of the matrices $\mathcal{L}^{11}, \mathcal{L}^{21}$, and $\mathcal{L}^{22}$ and the vector function $\beta$ are given by

$$
\begin{aligned}
\mathcal{L}_{j k}^{11}= & \frac{1}{\sqrt{R}}\left[\mathbf{Q}_{u}^{j \top} \mathcal{G}\left(\mathcal{L}^{\Delta} \mathbf{Q}_{u}^{k}\right)+\mathbf{Q}_{w}^{j \top} \mathcal{G}\left(\mathcal{L}^{\Delta} \mathbf{Q}_{w}^{k}\right)\right] \\
& +\left[\mathbf{Q}_{u}^{j} \mathcal{G}\left(\mathcal{L}^{\partial_{x}} \boldsymbol{Q}_{P}^{k}\right)+\mathbf{Q}_{w}^{j} \mathcal{G}\left(\mathcal{L}^{\partial_{y}} \boldsymbol{Q}_{P}^{k}\right)\right], \\
\mathcal{L}_{j k}^{12}= & \mathbf{Q}_{w}^{j \top} \mathcal{G} \mathbf{Q}_{\theta}^{k}, \quad \mathcal{L}_{j k}^{21}=\boldsymbol{Q}_{\theta}^{j \top} \mathcal{G} \mathbf{Q}_{w}^{k}, \quad \mathcal{L}_{j k}^{22}=\frac{1}{\sqrt{R}} \mathbf{Q}_{\theta}^{j \top} \mathcal{G}\left(\mathcal{L}^{\Delta} \boldsymbol{Q}_{\theta}^{k}\right), \\
\beta_{j}= & \mathbf{Q}_{\theta}^{j \top} \mathcal{G}\left[\boldsymbol{u}^{\mathrm{GS}} \cdot *\left(\mathcal{L}^{\partial_{x}} \boldsymbol{\theta}^{\mathrm{GS}}\right)+\boldsymbol{w}^{\mathrm{GS}} \cdot *\left(\mathcal{L}^{\partial_{z}} \boldsymbol{\theta}^{\mathrm{GS}}\right)\right] .
\end{aligned}
$$

Here, $\mathcal{L}^{\Delta}, \mathcal{L}^{\partial_{x}}$, and $\mathcal{L}^{\partial_{z}}$ are the collocation matrices associated with the operators $\Delta, \partial_{x}$, and $\partial_{z}$, respectively, $\boldsymbol{u}^{\mathrm{GS}}, \boldsymbol{w}^{\mathrm{GS}}$, and $\boldsymbol{\theta}^{\mathrm{GS}}$ are the expansions (46) and the operator * denotes componentby-component multiplication. Note that:

- The coefficient of $\mathbf{B}$ in Eq. (47) is the unit matrix because the thermal modes are orthonormal.

- Neither the continuity equation nor the boundary conditions have been used. This is because these linear homogeneous constraints are identically satisfied by the snapshots and thus by the POD modes (which are linear combinations of the snapshots) as well.

- The matrices $\mathcal{L}^{i j}$ are such that

$$
\left(\mathcal{L}^{11}\right)^{\top}=\mathcal{L}^{11}, \quad\left(\mathcal{L}^{12}\right)^{\top}=\mathcal{L}^{21}, \quad\left(\mathcal{L}^{22}\right)^{\top}=\mathcal{L}^{22} .
$$

- Also, because the hydrodynamic modes satisfy the continuity equation, whose discrete version is

$$
\mathcal{L}^{\partial_{x}} \mathbf{Q}_{u}^{j}+\mathcal{L}^{\partial_{z}} \mathbf{Q}_{w}^{j}=0
$$

the second term in the expression of $\mathcal{L}_{j k}^{11}$, i.e. the pressure term, identically vanishes, since

$$
\mathbf{Q}_{u}^{j} \mathcal{G}\left(\mathcal{L}^{\partial_{x}} \mathbf{Q}_{P}^{k}\right)+Q_{w}^{j} \mathcal{G}\left(\mathcal{L}^{\partial_{y}} \mathbf{Q}_{P}^{k}\right)=\left(\mathcal{L}^{\partial_{x}} \mathbf{Q}_{u}^{j}+\mathcal{L}^{\partial_{z}} \mathbf{Q}_{w}^{j}\right)^{\top} Q_{P}^{k}=0,
$$

where the second expression follows using the finitedimensional version of the identity

$$
\int_{\Omega} \boldsymbol{v} \cdot \nabla P d x d z=-\int_{\Omega} P \nabla \cdot \boldsymbol{v} d x d z=0
$$

which is readily obtained integrating by parts and taking into account that $\boldsymbol{v}=\mathbf{0}$ at the boundary.

\subsection{A symmetry preserving Galerkin system}

In principle, the GS (47) ignores the reflection symmetry (12). Let us now obtain a GS that preserves this symmetry. To this end, the POD modes are recalculated as explained in Section 3.3, which yields POD modes that are either reflection symmetric or antisymmetric, for both the hydrodynamic and thermal modes. In other words, the expansions (46) are replaced by

$$
\begin{gathered}
(\boldsymbol{u}, \boldsymbol{w}, \boldsymbol{P}) \simeq\left(\boldsymbol{u}^{\mathrm{GS}}, \boldsymbol{w}^{\mathrm{GS}}, \boldsymbol{P}^{\mathrm{GS}}\right)=\sum_{j=1}^{J_{v}^{+}} A_{j}^{+}\left(\mathbf{Q}_{u}^{j}, \mathbf{Q}_{w}^{j}, \mathbf{Q}_{\mathrm{P}}^{j}\right)^{+} \\
+\sum_{j=1}^{J_{v}^{-}} A_{j}^{-}\left(\mathbf{Q}_{u}^{j}, \mathbf{Q}_{w}^{j}, \mathbf{Q}_{\mathrm{P}}^{j}\right)^{-}, \\
\boldsymbol{\theta} \simeq \theta^{\mathrm{GS}}=\sum_{j=1}^{J_{\theta}^{+}} B_{j}^{+} \mathbf{Q}_{\theta}^{j+}+\sum_{j=1}^{J_{\theta}^{-}} B_{j}^{-} \mathbf{Q}_{\theta}^{j-} .
\end{gathered}
$$

Using these, the hydrodynamic and thermal amplitudes appearing in (47) are rewritten as
$\mathbf{A}=\left[\begin{array}{l}\mathbf{A}^{+} \\ \mathbf{A}^{-}\end{array}\right], \quad \mathbf{B}=\left[\begin{array}{l}\mathbf{B}^{+} \\ \mathbf{B}^{-}\end{array}\right]$

and, consistently with (12), the GS (47) becomes invariant under the reflection symmetry

$\left(\mathbf{A}^{+}, \mathbf{A}^{-}\right) \rightarrow\left(\mathbf{A}^{+},-\mathbf{A}^{-}\right), \quad\left(\mathbf{B}^{+}, \mathbf{B}^{-}\right) \rightarrow\left(\mathbf{B}^{+},-\mathbf{B}^{-}\right)$.

\subsection{Calculation of the bifurcation diagram using the Galerkin system}

Once the GS has been calculated, either taking the reflection symmetry into account or not, it can be used to calculate the bifurcation diagrams autonomously, namely not relying any more on the original governing equations (6)-(10), which are only used in the preprocess to calculate sets of snapshots.

The GS (47) is a system of differential algebraic equations (DAEs) that can be integrated numerically to obtain time dependent solutions. Also, the steady states are given by the system of nonlinear equations

$\mathcal{L}^{11} \mathbf{A}+\mathcal{L}^{12} \mathbf{B}=0, \quad \mathcal{L}^{21} \mathbf{A}+\mathcal{L}^{22} \mathbf{B}-\boldsymbol{\beta}(\mathbf{A}, \mathbf{B})=0$.

In particular, the conductive state is

$\mathbf{A}=\mathbf{0}, \quad \mathbf{B}=\mathbf{0}$.

The stability of a steady state is analyzed solving the linear eigenvalue problem

$\mathcal{L}^{11} \widetilde{\mathbf{A}}+\mathcal{L}^{12} \widetilde{\mathbf{B}}=0, \quad \widetilde{\mathcal{L}}^{21}(\mathbf{B}) \widetilde{\mathbf{A}}+\widetilde{\mathcal{L}}^{22}(\mathbf{A}) \widetilde{\mathbf{B}}=\lambda \widetilde{\mathbf{B}}$

Here, the matrices $\widetilde{\mathcal{L}}^{21}$ and $\widetilde{\mathcal{L}}^{21}$ depend on $\mathbf{A}$ and $\mathbf{B}$ and are given by $\widetilde{\mathcal{L}}^{21}=\mathcal{L}^{21}-\mathcal{L}_{\boldsymbol{\beta}}^{1}(\mathbf{B}), \quad \widetilde{\mathcal{L}}^{22}=\mathcal{L}^{22}-\mathcal{L}_{\beta}^{2}(\mathbf{A})$,

where the matrices $\mathcal{L}_{\beta}^{1}$ and $\mathcal{L}_{\beta}^{2}$ are the associated with the operators

$\widetilde{\mathbf{A}} \rightarrow \mathcal{L}_{\beta}^{1} \widetilde{\mathbf{A}} \equiv \boldsymbol{\beta}(\widetilde{\mathbf{A}}, \mathbf{B}), \quad \widetilde{\mathbf{B}} \rightarrow \mathcal{L}_{\beta}^{2} \widetilde{\mathbf{B}} \equiv \boldsymbol{\beta}(\mathbf{A}, \widetilde{\mathbf{B}})$.

For the conductive state (60), these matrices are not needed because

$\widetilde{\mathcal{L}}^{21}=\mathcal{L}^{21}, \quad \widetilde{\mathcal{L}}^{22}=\mathcal{L}^{22}$.

The bifurcation diagram could be calculated using pseudoarchlength continuation [1]. Here, instead, continuation will be performed as in [33], which will facilitate comparison of the computational efforts associated with the Lengendre collocation method and the GS. Specifically, continuation is based on the Newton method that, at each step, corrects the hydrodynamic and thermal amplitude vectors as

$(\mathbf{A}, \mathbf{B}) \rightarrow(\mathbf{A}+\widetilde{\mathbf{A}}, \mathbf{B}+\widetilde{\mathbf{B}})$,

where the corrections are calculated solving the following linear system

$\mathcal{L}^{11} \widetilde{\mathbf{A}}+\mathcal{L}^{12} \widetilde{\mathbf{B}}=-\left[\mathcal{L}^{11} \mathbf{A}+\mathcal{L}^{12} \mathbf{B}\right]$,

$\widetilde{\mathcal{L}}^{21}(\mathbf{B}) \widetilde{\mathbf{A}}+\widetilde{\mathcal{L}}^{22}(\mathbf{A}) \widetilde{\mathbf{B}}=-\left[\mathcal{L}^{21} \mathbf{A}+\mathcal{L}^{22} \mathbf{B}-\boldsymbol{\beta}(\mathbf{A}, \mathbf{B})\right]$,

with the matrices $\mathcal{L}^{1 j}$ and $\widetilde{\mathcal{L}}^{2 j}$ as given by (48), (49) and (62). It must be noted here that the matrices $\mathcal{L}^{j k}$ may be calculated from the outset (at each $R$ ) in the iteration procedure, but the bilinear vector function $\beta$ and the matrices $\widetilde{\mathcal{L}}^{21}$ and $\widetilde{\mathcal{L}}^{22}$ must be calculated reconstructing (at all collocation points) the velocity and temperature fields in the previous iteration step. This is the most computationally expensive part in the calculation, and could be improved either using an inner product based on fewer grid points or taking advantage of the fact that $\beta$ is bilinear and writing its components as $\beta_{j}(\mathbf{A}, \mathbf{B})=\sum_{k, l} b_{j k} A_{k} B_{l}$, where the coefficients $\beta_{j k l}$ can be calculated in the preprocess using, e.g., a discrete empirical interpolation 
method [12]. But these improvements are outside the scope of this paper.

The system (66) and (67) is solved for values of the Rayleigh number $R$ in the interval $1000 \leqslant R \leqslant 3000$ to construct the bifurcation diagram using the POD modes, taking the symmetry into account or not. The process is performed starting with a known solution from the Legendre solver at a value of the Rayleigh number $R=1000$ and increasing $R$ in small steps. At each new value of $R$, the initial iterate for the Newton method is the steady state for the former value of $R$, except near the bifurcation points, which require specific treatment. The method proceeds in six steps, as follows:

1. To begin with, the stability of the conductive branch $(60)$ and threshold values of the Rayleigh number at the bifurcation points in this branch are analyzed by solving the (generalized) eigenvalue problem (61), with the coefficient matrices as given by (64). In particular, the threshold values of $R, R_{c}$, are calculated (via, e.g., the secant method) from the scalar equation

$$
\operatorname{det} \mathcal{L}=0, \quad \text { with } \mathcal{L}=\left[\mathcal{L}^{11}, \mathcal{L}^{12} ; \mathcal{L}^{21}, \mathcal{L}^{22}\right] .
$$

Note that because of (51), the matrix $\mathcal{L}$ is symmetric, which means that the eigenvalues of (61) are all real for the conductive state, which is consistent with the similar property for the governing equations, mentioned after the formulation (16)-(20). Eq. (68) provides the thresholds $R_{c}^{1}$ and $R_{c}^{2}$ in Fig. 2.

2. At each bifurcation point, the bifurcated branches are initiated (for $R$ both slightly smaller and larger than $R_{c}$ ) using the Newton method described above with an initial iterate

$$
(\mathbf{A}, \mathbf{B})=\alpha(\widetilde{\mathbf{A}}, \widetilde{\mathbf{B}}),
$$

where $(\widetilde{\mathbf{A}}, \widetilde{\mathbf{B}})$ is an eigenvector of (61) associated with $\lambda=0$. This must be performed for both $\alpha>0$ and $\alpha<0$, with $|\alpha|$ conveniently small, but not too small, to avoid decaying to the conductive state (60). Near, e.g., the pitchfork bifurcation at $R=R_{c}^{1}$ in Fig. 2, one point is obtained at each of the bifurcated branches for $R>R_{c}^{1}$ and nothing new for $R<R_{c}^{1}$. The same behaviour is observed in the pitchfork bifurcation at $R=R_{c}^{2}$. It is to be noted that this Newton iteration can be very slow if $R$ is too close to $R_{c}^{1}$ and $R_{c}^{2}$.

3. The continuation process is performed as follows: we start with a known solution from the Legendre solver at a value of the Rayleigh number $R_{1}$. Once the system is solved, the solution at $R_{2}$ is obtained by solving (66) and (67) with initial condition obtained in the previous step. Therefore, the process continues solving the linear system at $R_{j}$ from the solution at $R_{j-1}$.

4. The secondary pitchfork bifurcations at the solutions branches are identified using the counterpart of (68) for general, non-conductive states, namely

$$
\text { det } \widetilde{\mathcal{L}}=0, \quad \text { with } \widetilde{\mathcal{L}}=\left[\mathcal{L}^{11}, \mathcal{L}^{12} ; \widetilde{\mathcal{L}}^{21}(\mathbf{B}), \widetilde{\mathcal{L}}^{22}(\mathbf{A})\right]
$$

Thus, the sign of the left hand side of (70) can be monitored along the solution branches and note that a change in this sign indicates that a bifurcation point is nearby. Precise calculation of the new bifurcation points is performed solving (70) with the secant method. This identifies in Fig. 2 the two pitchfork bifurcations that occur at $R=R_{\mathrm{c}}^{3}$.

5. The new branches emanating at these bifurcation points are continued numerically as explained in steps 2 and 3 above. This provides the new bifurcated branches that appear in Fig. 2 at $R=R_{c}^{3}$.

6. The process continues identifying new bifurcation points in the new branches and continuing along the branches as explained above.

\section{Results for the thermal convection problem}

The GSs described in Section 4 are constructed for the thermal convection problem (6)-(10) as follows. The POD modes calculated from snapshots calculated at $R=1000$ and 1500 , as explained in Section 3 , by the time-dependent solver (to be called temporal snapshots below) will be first used to construct the GS in Section 5.1, while new POD modes resulting from snapshots computed by a Newton method (to be called Newton snapshots) at the same values of $R$ will be considered in Section 5.2. In both cases, according to our discussion in Section 3 and 4, four ROMs will be considered, namely:

- Unsymmetric ROM (see Section 4.1), using the modes calculated as explained in Section 3.2, which do not take the symmetries into account.

- Symmetry-preserving ROM (see Section 4.2), using the POD modes calculated as explained in Section 3.3, which takes the horizontal reflection symmetry into account.

Each of these ROMs will use some snapshots calculated at either $R=1000$ or $R=1500$. In all cases, the numbers of retained hydrodynamic and thermal modes ( $n_{v}$ and $n_{\theta}$, respectively) will be chosen after some calibration on the resulting bifurcation diagrams, beginning with the numbers of POD modes selected in Section 3.

Concerning the computational cost, the full spectral Legendre collocation solver with an expansion of $17 \times 13$ modes involves $18 \times 14$ collocation points. Since the system involves four scalar flow variables, the total number of unknowns and equations is $4 \times 18 \times 14$. Typical numerical tasks such as simulating time dependent runs or using a Newton solver to calculate steady states require solving linear iterations which in turn require calculating residuals. Taking into account the tensorial nature of the spectral decomposition, calculating residuals involves a computational effort that scales at worse as $\mathcal{O}\left(4 \times 18^{2} \times 14\right.$ ) (see, e.g. $[9,11]$ ). The residuals of the GS retaining $n_{v}$ hydrodynamic modes and $n_{\theta}$ thermal modes, using the whole $18 \times 14$-mesh to perform Galerkin projection involves a computational effort that scales with $18 \times 14 \times\left(n_{v}+n_{\theta}\right)$. Therefore, the ratio of the CPU times required by the full Legendre solver and the GS scales with $4 \times 18^{2} /\left(n_{v}+n_{\theta}\right)$, which means that the acceleration factor will not be quite large, except for small $n_{v}+n_{\theta}$. But it must be kept in mind that this ratio can be highly decreased using a fewer amount of mesh points to perform the Galerkin projection, which will be done elsewhere. In any event, comparing with other reduced methods, the offine time is much less expensive in this case because snapshots are calculated for just one value of the Rayleigh number. For instance, according to Table 1 below, the total (offline + online) CPU time required to calculate the bifurcation diagram with the present method is aproximately 2 (in general less than 2) CPU minutes, while the time required by Legendre solver is $8.744 \mathrm{CPU}$ minutes. The offline CPU time, which is what the new ideas in the paper try to decrease by calculating the snapshots at just one value of $R$, is only $O\left(10^{-2}\right)$ minutes.

\section{Table 1}

Hydrodynamic $\left(n_{v}\right)$ and thermal $\left(n_{\theta}\right)$ retained modes and (offline + online) CPU time (in minutes) required to calculate the bifurcation diagram by the unsymmetric and

\begin{tabular}{|c|c|c|c|c|c|c|}
\hline \multirow[t]{2}{*}{ Snapshots calculated at } & \multicolumn{3}{|c|}{$R=1000$} & \multicolumn{3}{|c|}{$R=1500$} \\
\hline & $n_{v}$ & $n_{\theta}$ & $\mathrm{CPU}$ & $n_{v}$ & $n_{\theta}$ & $\mathrm{CPU}$ \\
\hline Unsymmetric ROM & 7 & 9 & 1.855 & 9 & 11 & 1.959 \\
\hline Symm. preser. ROM & $5+5$ & $5+5$ & 1.901 & $7+7$ & $10+12$ & 2.120 \\
\hline
\end{tabular}
symmetry preserving ROMs based on the temporal POD snapshots calculated at $R=1000$ and 1500 , as indicated. 

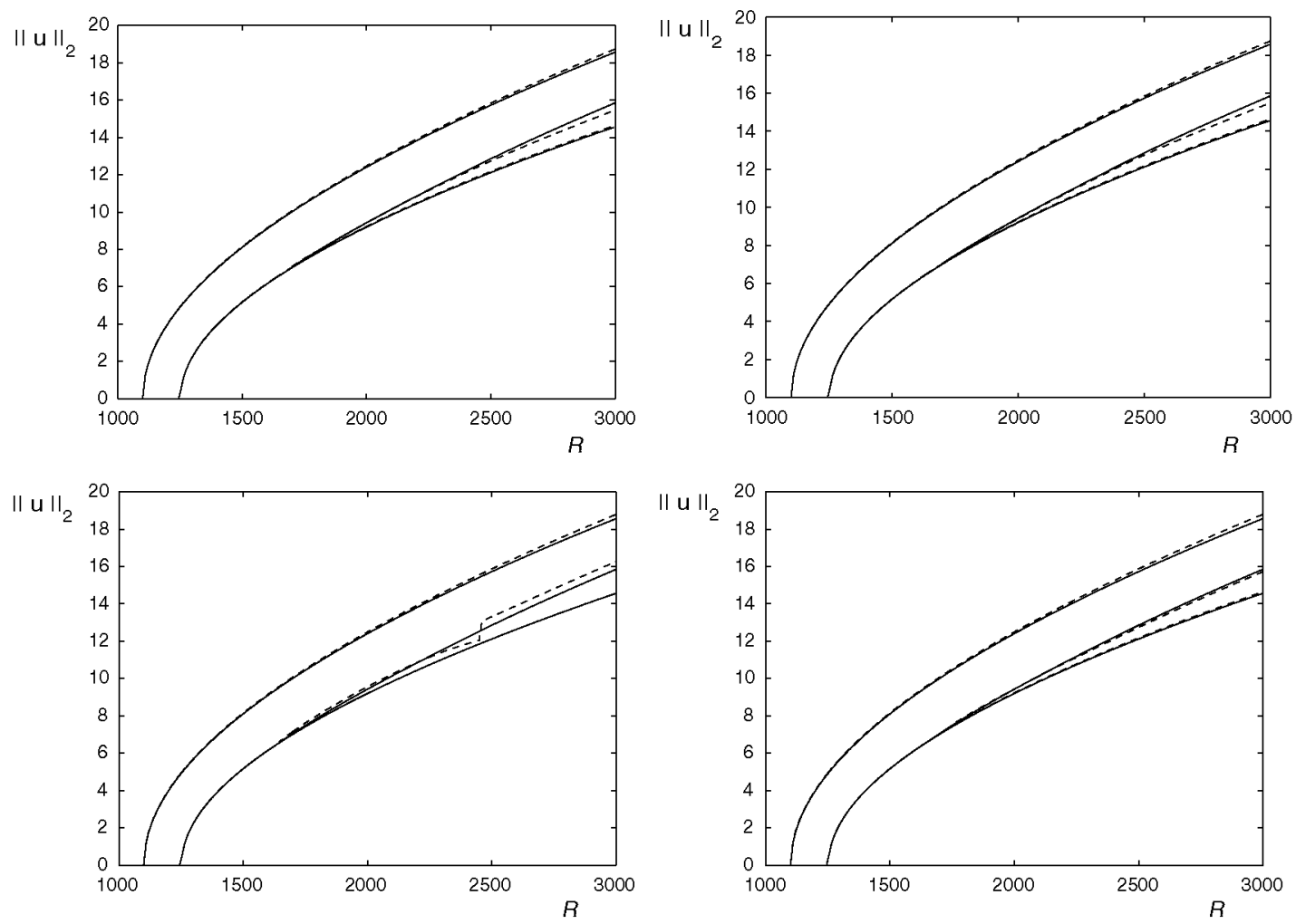

Fig. 6. Bifurcation diagrams for (6)-(10), as calculated using the full Legendre collocation method (solid lines) and the RoMs (dashed lines), considering the unsymmetric ROM (left) and the reflection symmetric ROM (right), based on temporal POD modes calculated at $R=1000$ (top) and 1500 (bottom).
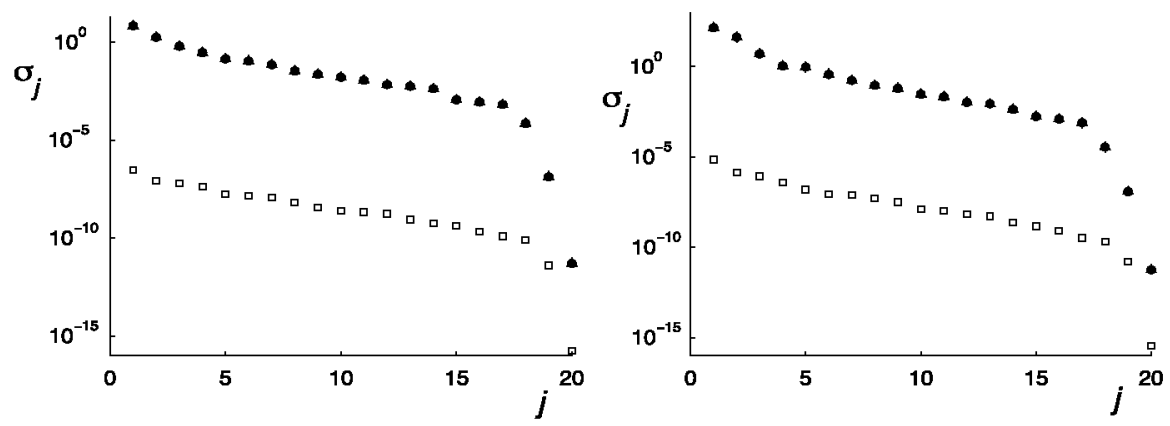

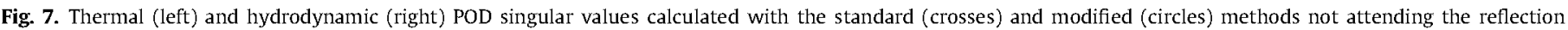

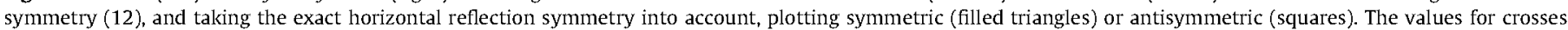
circles and triangles are plot indistinguishable.
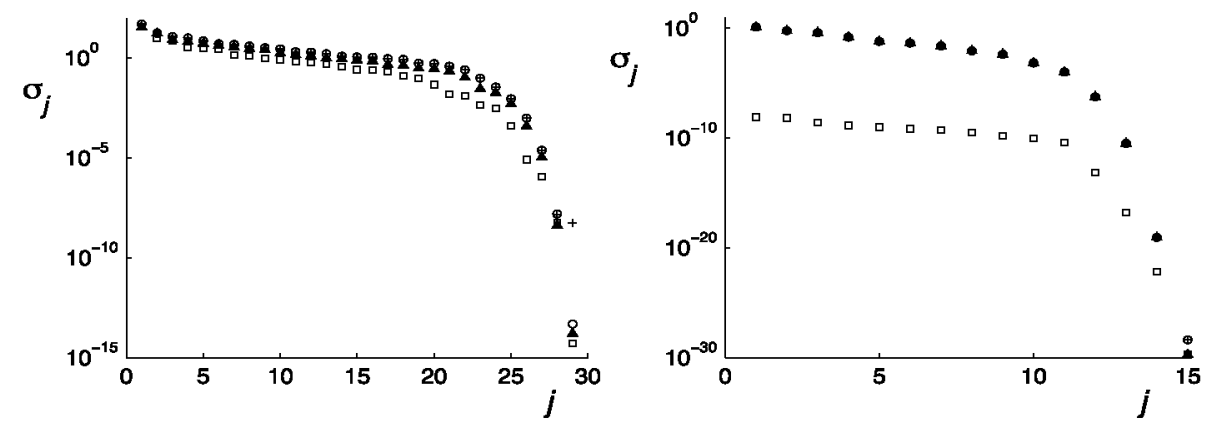

Fig. 8. Counterparts of Fig. 7-left using the Newton thermal snapshots at $R=1500$ near the convective (left) and conductive (right) states. 
Table 2

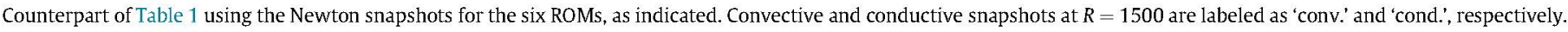

\begin{tabular}{|c|c|c|c|c|c|c|c|c|c|}
\hline \multirow[t]{2}{*}{ Snapshots at $R=$} & \multicolumn{3}{|l|}{1000} & \multicolumn{3}{|c|}{1500 conv. } & \multicolumn{3}{|c|}{1500 cond. } \\
\hline & $n_{v}$ & $n_{\theta}$ & CPU & $n_{\nu}$ & $n_{\theta}$ & CPU & $n_{v}$ & $n_{\theta}$ & CPU \\
\hline Unsymmetric ROM & 4 & 2 & 1.708 & 4 & 5 & 1.748 & 3 & 3 & 1.692 \\
\hline Symm. preser. ROM & $4+4$ & $2+2$ & 1.754 & $4+4$ & $5+5$ & 1.787 & $3+3$ & $3+3$ & 1.793 \\
\hline
\end{tabular}

\subsection{Galerkin systems based on temporal modes}

Let us now consider the temporal snapshots calculated by the time-dependent solver and apply the two above mentioned ROMs. Using (30) and (45) for the unsymmetric and symmetry-preserving ROMs, respectively, the numbers of the hydrodynamic and thermal modes are summarized in Table 1. The larger number modes at $R=1500$ is due to the fact that the dynamics near the non-conductive state is richer. The required CPU times using a $3.06 \mathrm{GHz}$ Intel microprocessor are also given in Table 1. Note that the ROMs overperform the Legendre solver (which requires $8.744 \mathrm{CPU}$ minutes) by a factor larger than 4 . The symmetric and unsymmetric ROMs considered in Table 1
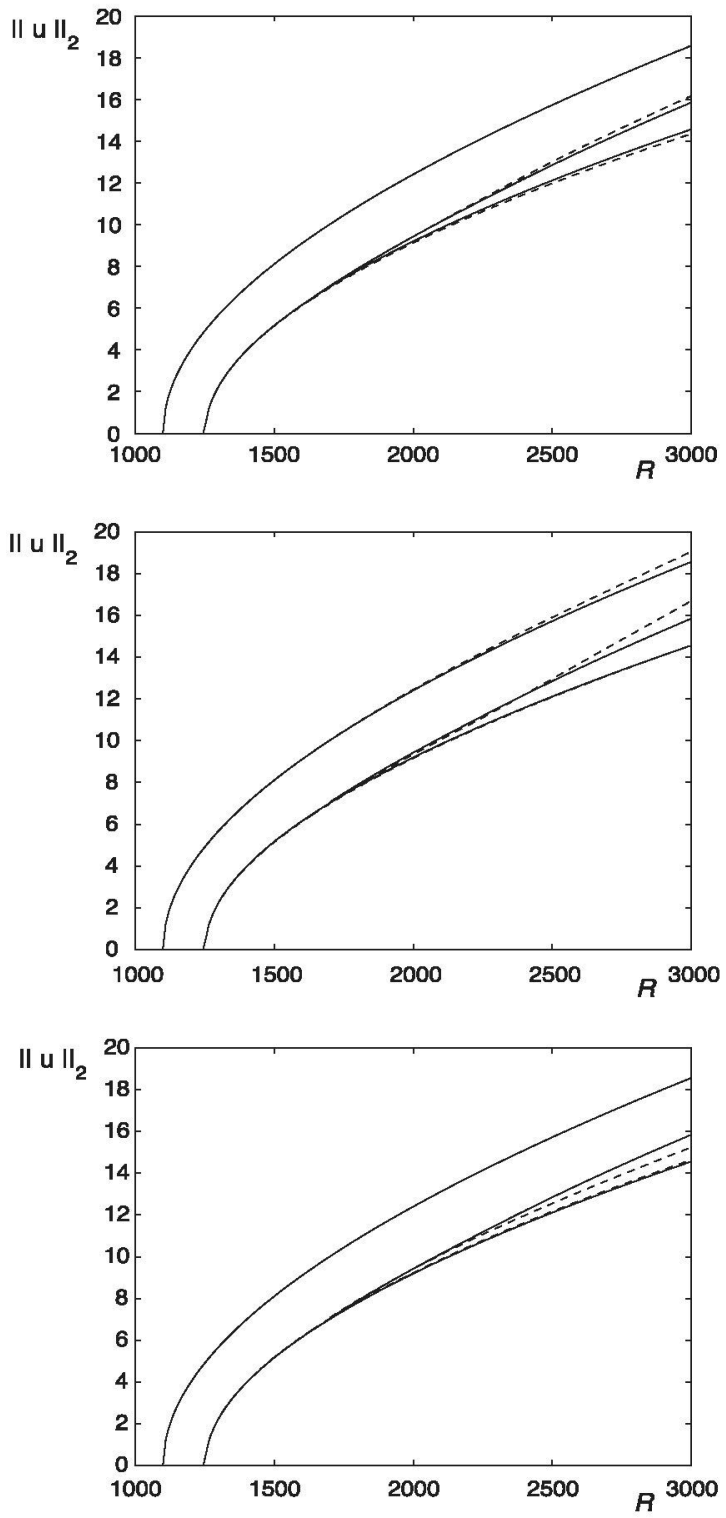

produce the bifurcation diagrams given in Fig. 6, where the $L^{2}$-norm of the horizontal velocity component (21) is plotted vs. $R$. As can be seen in this figure:

- In all cases, the ROMs produce bifurcation diagrams that approximate that computed by the Legendre solver within a $O\left(10^{-1}\right)$-error in the whole interval. This error is only of the order of $O\left(10^{-4}\right)$ near the values of the Rayleigh number where the snapshots were calculated (namely, at $R=1000$ and 1500) and smoothly worsens as the Rayleigh number is increased.

- With the snapshots calculated at $R=1000$, the unsymmetric and the symmetry-preserving ROMs produce similar results.
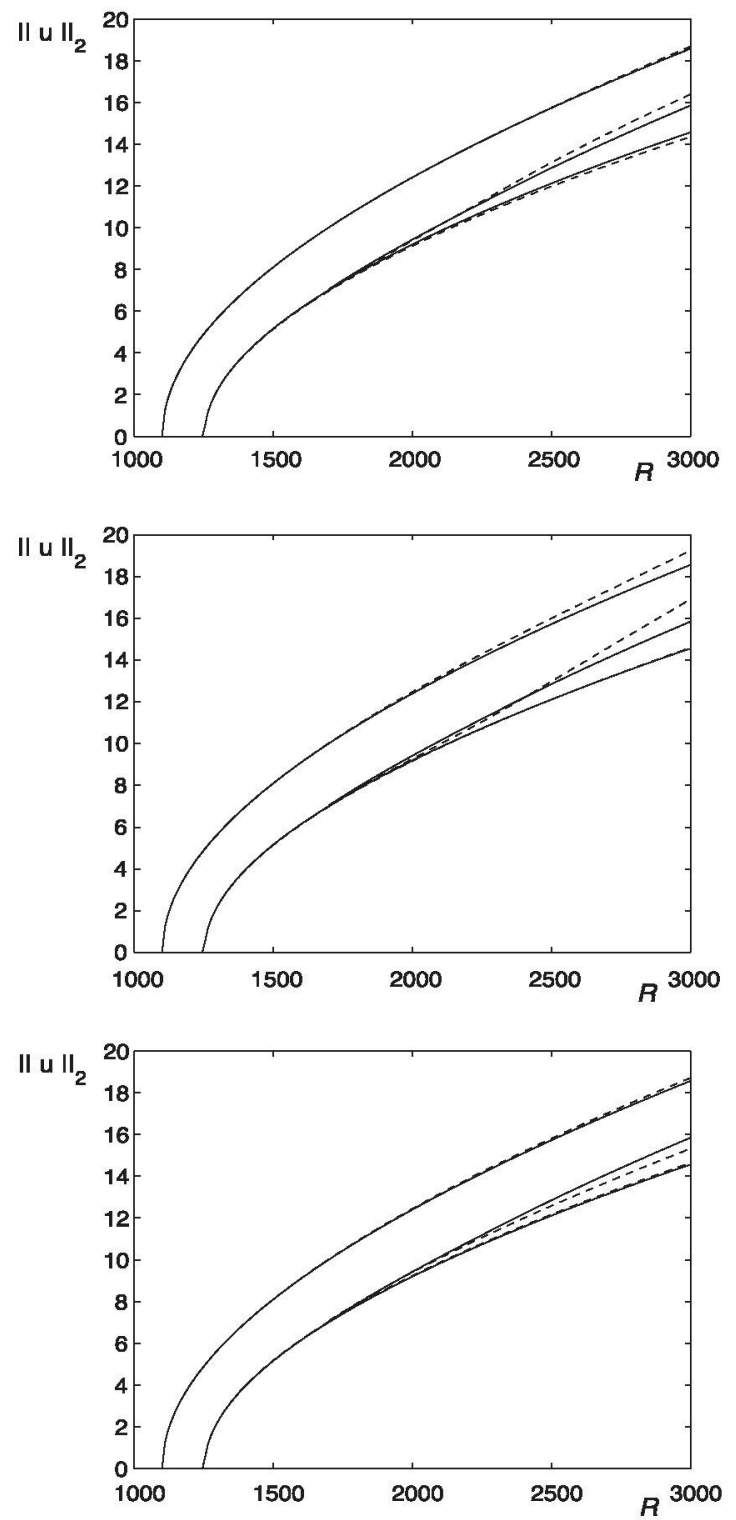

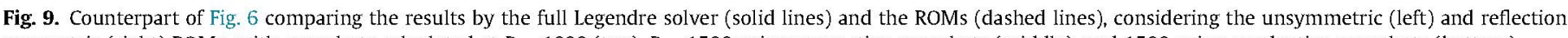
symmetric (right) RoMs, with snapshots calculated at $R=1000$ (top), $R=1500$ using convective snapshots (middle), and 1500 using conductive snapshots (bottom). 
- With the snapshots calculated at $R=1500$, instead, the symmetry-preserving ROM clearly improves the approximation by the unsymmetric ROM.

\subsection{Galerkin systems based on Newton modes}

Now, we take as snapshots the various Newton iterations required to calculate steady states with initial conditions as defined in Eq. (22). In fact, we take three sets of snapshots, namely:

- One set of snapshots obtained at $R=1000$ taking initial conditions close to the conductive state. The associated unsymmetric, symmetric, and antisymmetric singular values are plotted in Fig. 7. Note that, as with temporal snapshots, thermal and hydrodynamic modes exhibit similar trends.

- Two sets of snapshots at $R=1500$, one taking initial conditions close to the convective state (to be referred to below as convective snapshots) and another with initial conditions close to the conductive state (conductive snapshots). Note that latter snapshots could not be obtained with the temporal solver because the conductive state is unstable at $R=1500$; the Newton method, instead, does converge also to unstable steady states. The associated singular values are similar to those obtained at $R=1000$, as illustrated for thermal snapshots in Fig. 8 .

Figs. 7 and 8 show that the number of useful modes is now smaller, which is due to the fact that Newton iterations converge faster than the temporal runs. In fact, the Newton method requires 20 iterations to reach convergence at $R=1000$, while at $R=1500$ the number of iterations is 30 and 15 for convective and conductive initial conditions, respectively.

For each set of snapshots, both the unsymmetric and symmetry-preserving ROMs will be constructed, meaning that six different GSs will be considered. Proceeding with the construction of the GS, Eqs. (30) and (45) are used to fix the number of retained modes for the unsymmetric and symmetry-preserving ROMs, respectively, which gives the numbers of modes summarized in Table 2. As anticipated, the numbers of retained modes are smaller than when temporal snapshots were used. The numbers of modes in the symmetry-preserving case doubles their counterparts for the unsymmetric ROMs. Thus, the ability of the former ROM to extract more information from the snapshots is even clearer than when temporal modes were used. Table 2 also shows that the number of useful modes is much larger when the snapshots result from the perturbed dynamics of the bifurcated state at $R=1500$ than when they result from perturbations of the conductive state, at $R=1000$ or $R=1500$ conductive. This is because the dynamics near the non-conductive state is richer. Using a $3.06 \mathrm{GHz}$ Intel microprocessor, the total (offline + online) CPU times (in minutes) required by each ROM is also indicated in Table 2 and shows that this time is slightly smaller than when temporal snapshots were used. Again, the ROMs overperform the full Legendre solver (which requires $8.744 \mathrm{CPU}$ minutes) by a factor larger than $8.744 / 1.793>4.8$.

The six ROMs considered in this subsection produce the bifurcation diagrams plotted in Fig. 9, which are very similar to those in Fig. 6. As can be seen:

- The difference between the Legendre and POD bifurcation diagrams is less than $O\left(10^{-7}\right)$ near the values of $R$ where the snapshots are calculated, and smoothly worsens as $R$ is increased, especially after the secondary inverse Pithcfork bifurcation.

- The conductive snapshots at $R=1000$ and $R=1500$ provide a fairly good approximation, both for the unsymmetric and symmetry preserving ROMs.

\section{Concluding remarks}

A Galerkin method based on POD has been presented to construct the bifurcation diagram giving the steady states, as the Rayleigh number is varied, in a two-dimensional RayleighBénard convection problem for large Prandtl number in a rectangular box. Because of an appropriate combination of slip/noslip boundary conditions, this problem exhibits a horizontal reflection symmetry. The POD modes have been obtained from snapshots computed by the Legendre solver at just one value of the Rayleigh number. The snapshots have been calculated as unconverged states of iterates converging to a steady state (either a conductive or convective steady state), either using a time-dependent solver or a Newton method. Maximizing the information contained in these unconverged snapshots has required using an improved version of the standard method to calculate the POD modes. The modified method provides good approximations of the POD modes associated with very small singular values. In addition, the POD modes have been obtained in two ways, namely ignoring the horizontal reflection symmetry or taking this symmetry into account.

Using POD modes calculated in the various above mentioned ways, several Galerkin systems have been obtained that were combined with a basic continuation method to compute the bifurcation diagram in the Rayleigh number range $1000 \leq R \leq 3000$. As general conclusions from this analysis, it has been shown that:

- Calculating the snapshots from outcomes of the Legendre solver at just one value of the bifurcation parameter means that the computational cost of the preprocess is very small. In fact, the whole computational cost (proprocess + online operation) of the method is at least four times smaller than that the Legendre solver.

- The specific value of the Rayleigh number where snapshots are calculated is not critical, but it can be chosen with great flexibility.

- The number of snapshots calculated by the time-dependent solver is larger than when using the Newton method.

- The reflection symmetry allows for extracting a larger number of useful POD modes from a set of un-symmetric snapshots.

- The approximated bifurcation diagram calculated with the various Galerkin systems is reasonably good. Departure from its counterpart calculated by the full Legendre solver is less than $O\left(10^{-7}\right)$ near the values of the Rayleigh number where the snapshots were calculated and it smoothly worsens as the Rayleigh number is increased.

The performance of the ROM could be improved in various ways. On the one hand, a more efficient projection of the nonlinear terms onto the POD modes, which has been made here upon reconstruction of the nonlinear terms and projection using the standard $L^{2}$-inner product, which involves a fairly large computational cost to project the nonlinear terms. Instead, the Galerkin projection can be performed using a scalar product based on a few amount of points [39,51] or the so-called discrete empirical interpolation [12] can be used. Also, an adaptive method can be used to combine on demand the low-dimensional ROM system and the Legendre numerical solver. None on these improvements have been used here, where the main focus has been to illustrate how the usual way to calculate snapshots in the preprocess can be substituted by a more flexible and computational inexpensive method. Any improvement in the online operation of the ROM would further increase its overall performance. 


\section{Acknowledgements}

This work was partially supported by MINECO (Spanish Government), under Grant MTM2012-37642, which includes RDEF funds, and TRA2013-45808-R. The authors thank Dr. Rapún for a critical reading of the manuscript. The authors are indebted to two anonymous referees for several useful comments and suggestions on an earlier version, which helped to improve the paper.

\section{References}

[1] Allgower EL, Georg K. Introduction to numerical continuation methods. SIAM classics in applied mathematics, vol. 45. SIAM; 2003.

[2] Alonso D, Velazquez A, Vega JM. A method to generate computationally efficient reduced order models. Comput Meth Appl Mech Eng 2009;198:2683-91.

[3] Alonso D, Vega JM, Velazquez A. Reduced order model for viscous aerodynamic flow past an aitfoil. AIAA J 2010;48:1946-58.

[4] Alonso D, Vega JM, Velazquez A, de Pablo V. Reduced-order modeling of threedimensional extemal aerodynamic flows. J Aerospace Eng 2012;25:588-99.

[5] Aranson IS, Kramer L. The world of the complex Ginzburg-Landau equation. Rev Mod Phys 2002; 74:100-42.

[6] Aubry N, Lyan WY, Titi ES. Preserving symmetries in the proper orthogonal decomposition. SIAM J Sci Comput 1993;14:483-505.

[7] Bache E, Vega JM, Velazquez A. Model reduction in the back step fluid-thermal problem with variable geometry. Int J Thermal Sci 2010:49:2376-84.

[8] Bénard H. Les tourbillons cèllulaires dans une nappe liquide. Rev Gen Sci Pures Appl Bull Assoc 1900;11:1261-71.

[9] Bernardi C, Maday Y. Approximations spectrales de problèmes aux limites elliptiques. Springer-Verlag; 1991.

[10] Braconnier T, Ferrier M, Jouhaud JC, Montagnac M, Sagaut P. Towards an adaptive POD/SVD surrogate model for aeronautic design. Comput Fluids 2011;40:195-209.

[11] Canuto C, Hussaini MY, Quarteroni A, Zang TA. Spectral methods in fluid dynamics. New York: Springer-Verlag; 1988.

[12] Chaturantabut $S$, Sorensen DC. Nonlinear model reduction via discrete empirical interpolation. SIAM J Sci Comput 2010;32(5):2737-64.

[13] Couplet M, Basdevant C, Sagaut P. Calibrated reduced-order POD-Galerkin system for fluid flow modelling. J Comput Phys 2005;207:192-220.

[14] Crawford JD, Knobloch E. Symmetry and symmetry-breaking bifurcations in fluid dynamics. Annu Rev Fluid Mech 1991;23:341-87.

[15] Davies GF. Dynamic earth, plates, plumes and mantle convection. Cambridge University Press; 2001.

[16] Deparis S, Rozza G. Reduced basis method for multi-parameter-dependent steady Navier-Stokes equations: applications to natural convection in a cavity. J Comput Phys 2009;228(12):4359-4378.

[17] Dowell EH, Hall KC. Modeling of fluid-structure interaction. Annu Rev Fluid Mech 2001;33:445-90.

[18] Foias C, Sell GR, Temam R. Inertial manifolds for nonlinear evolutionary equations. J Differ Eqations 1988;73:309-53.

[19] Foias C, Nicolaenko B, Sell GR, Temam R. Inertial manifolds for the KuramotoSivashinsky equation and an estimate of their lowest dimension. J Math Pures Appl 1988;67:197-226.

[20] Golub GH, Van Loan CF. Matrix computations. John Hopkins Univ Press: 1996.

[21] Golubitsky M, Swift JW, Knobloch E. Symmetries and pattern selection in Rayleigh-Bénard convection. Physica D 1984;10:249-76.

[22] Haragus M, Iooss G. Local bifurcations, center manifolds, and normal forms in infinite dimensional dynamical systems. Springer; 2010.

[23] Herrero H, Maday Y, Pla F. RB (Reduced Basis) applied to RB (Rayleigh-Bénard). Comput Methods Appl Mech Eng 2013;261-262:132-41.

[24] Ilak M, Bagheri S, Brandt L, Rowley CW, Henningson DS. Model reduction of the nonlinear complex Ginzburg-Landau equation. SIAM J Appl Dyn Syst 2010;9:1284-302.

[25] Jolly MS. Bifurcation computations on an approximate inertial manifold for the 2D Navier-Stokes equations. Physica D 1993;63:8-20.
[26] Kunisch K, Volkwein S. Galerkin proper orthogonal decomposition methods for a general equation in fluid dynamics. SIAM J Numer Anal 2003;40(2):492-515.

[27] Kutnetsov Y. Elements of applied bifurcation theory. Applied mathematical sciences, vol. 112. Springer; 2004.

[28] Lassila T, Manzoni A, Ouarteroni A, Rozza G. Model order reduction in fuid dynamics: challenges and perspectives. In: Quarteroni A, Rozza G, editors. Reduced order methods for modeling and computational reduction, vol. 9. Springer MS\&A Series; 2014. p. 235-74.

[29] LeGresley PA, Alonso JJ. Investigation of non-linear projection for POD based reduced order models for aerodynamics. AIAA paper 2001-0926. 39th AIAA aerospace sciences meeting \& exhibit; 2001.

[30] Lieu T, Farhat C, Lesoinne M. Reduced-order fluid/structure modeling of a complete aircraft configuration. Comput Meth Appl Mech Eng 2006; $195: 5730-42$.

[31] Lucia DJ, Beran PS, Silva WA. Reduced-order modelling: new approaches for computations physics. Prog Aerosp Sci 2004;40:51-117.

[32] Manzoni A. An efficient computational framework for reduced basis approximation and a posteriori error estimation of parametrized NavierStokes flows. ESAIM Math Model Numer Anal 2014;48:1199-226.

[33] Pla F, Mancho AM, Herrero H. Bifurcation phenomena in a convection problem with temperature dependent viscosity at low aspect ratio. Physica D $2008: 238: 572-80$

[34] Promislow K, Temam R. Localization and approximation of attractors for the Ginzburg-Landau equation. J Dyn Differ Equations 1991;3:491-514.

[35] Prud'homme C, Rovas DV, Veroy K, Machiels L, Maday Y, Patera AT, et al. Reliable real-time solution of parametrized partial differential equations: reduced-basis output bounds methods. J Fluids Eng 2002;124:70-80.

[36] Rapún ML, Vega JM. Reduced order models based on local POD plus Galerkin projection. J Comput Phys 2010;229:3046-63.

[37] Rempfer D. Low-dimensional modeling and numerical simulation of transition in simple shear flows. Annu Rev Fluid Mech 2003;35:229-65.

[38] Richter FM, Nataf HC, Daly SF. Heat transfer and horizontally averaged temperature of convection with large viscosity variations. J Fluid Mech $1983 ; 129: 173-92$

[39] Ryckelynck D. Hyper-reduction of mechanical models involving internal variables. Int I Numer Methods Eng 2009;77:75-89.

[40] Robinson JC. Computing inertial manifolds. Discrete Cont Dyn Syst $2002 ; 8: 815-33$.

[41] Rozza G, Huynh DBP, Patera AT. Reduced basis approximation and a posteriori error estimation for affinely parametrized elliptic coercive partial differential equations. Arch Comput Methods Eng 2008;15:229-75.

[42] Shah M, Sorensen DC. A symmetry preserving singular value decomposition. SIAM J Matrix Anal Appl 2006;28:749-69.

[43] Shah M. A symmetry preserving singular value decomposition. $\mathrm{PhD}$ Thesis. CAAM, Rice University, TR07-06; 2007.

[44] Shah M, Sorensen DC. Best non-spherical symmetric low rank approximation. SIAM J Matrix Anal Appl 2010;31:1019-39.

[45] Siegel SG, Seidel J, Fagley C, Luchtenburg DM, Cohen K, McLaughlin T. Lowdimensional modelling of a transient cylinder wake using double proper orthogonal decomposition. J Fluid Mech 2008;610:1-42.

[46] Sirisup S, Karniadakis GE. Stability and accuracy of periodic flow solutions obtained by a POD-penalty method. Physica D 2005;202:218-37.

[47] Sirisup S, Karniadakis GE, Xiu D, Kevrekidis IG. Equations-free/Galerkin-free POD assisted computation of incompressible flows. J Comput Phys 2005:207:568-87.

[48] Strikwerda JC. Finite difference schemes and partial differential. Pacific Grove (CA): Wadsworth and Brooks; 1989.

[49] Terragni F, Valero E, Vega JM. Local POD plus Galerkin projection in the unsteady lid-driven cavity problem. SIAM J Sci Comput 2011;33:3538-61.

[50] Terragni F, Vega JM. On the use of POD-based ROMs to analyze bifurcations in some dissipative systems. Physica D 2012:241:1393-405.

[51] Terragni F, Vega JM. Construction of bifurcation diagrams using POD on the fly. SIAM J Appl Dyn Syst 2014;13:330-65.

[52] Thomas JP, Dowell EH, Hall KC. Using automatic differentiation to create a nonlinear reduced-order-model aerodynamic solver. AlAA J 2010:48:19-24.

[53] Zienkiewicz OC. The finite element method in engineering science. McGrawHill; 1971. 